\title{
Sliding Mode Control of Two-Level Quantum Systems
}

\author{
Daoyi Dong and Ian R. Petersen
}

\begin{abstract}
This paper proposes a robust control method based on sliding mode design for two-level quantum systems with bounded uncertainties. An eigenstate of the two-level quantum system is identified as a sliding mode. The objective is to design a control law to steer the system's state into the sliding mode domain and then maintain it in that domain when bounded uncertainties exist in the system Hamiltonian. We propose a controller design method using the Lyapunov methodology and periodic projective measurements. In particular, we give conditions for designing such a control law, which can guarantee the desired robustness in the presence of the uncertainties. The sliding mode control method has potential applications to quantum information processing with uncertainties.

\section{Index Terms}

quantum control, sliding mode control, bounded uncertainty, periodic projective measurement, Lyapunov methodology.
\end{abstract}

\section{INTRODUCTION}

The manipulation and control of quantum systems is becoming an important task in many fields [1]-[3], such as atomic physics [4], molecular chemistry [5] and quantum information [6].

The material in this paper was partially presented at the 2010 American Control Conference at Baltimore, Maryland, USA. This work was supported by the Australian Research Council and in part by the National Natural Science Foundation of China under Grant 60703083 and Grant 60805029.

D. Dong is with the School of Engineering and Information Technology, University of New South Wales at the Australian Defence Force Academy, Canberra, ACT 2600, Australia and with the Institute of Cyber Systems and Control, State Key Laboratory of Industrial Control Technology, Zhejiang University, Hangzhou 310027, China daoyidong@gmail . com

I. R. Petersen is with the School of Engineering and Information Technology, University of New South Wales at the Australian Defence Force Academy, Canberra, ACT 2600, Australia i.r.petersen@gmail . com 
It is desirable to develop quantum control theory in a systematic way in order to adapt it to the development of quantum technology [7]. Several useful tools from classical control theory have been introduced to the control analysis and design of quantum systems. For example, optimal control theory has been used to assist in control design for closed and dissipative quantum systems [8]-[14]. A learning control method has been presented for guiding the control of chemical reactions [5], [15]. Quantum feedback control approaches including measurement-based feedback and coherent feedback have been used to improve performance for several classes of tasks such as preparing quantum states, quantum error correction, controlling quantum entanglement [16][30]. Robust control tools have been introduced to enhance the robustness of quantum feedback networks and linear quantum stochastic systems [31], [32].

Although some progress has been made, more research effort is necessary in controlling quantum phenomena. In particular, the robustness of quantum control systems has been recognized as a key issue in developing practical quantum technology [33]-[35]. In this paper, we focus on the robustness problem for quantum control systems. In [32], James and co-workers have formulated and solved a quantum robust control problem using the $H^{\infty}$ method for linear quantum stochastic systems. Here, we develop a variable structure control approach with sliding modes to enhance the robustness of quantum systems. The variable structure control strategy is a widely used design method in classical control theory and industrial applications where one can change the controller structure according to a specified switching logic in order to obtain desired closed-loop properties [36], [37]. In [38] and [39], Dong and Petersen have formulated and solved a variable structure control problem for the control of quantum systems. However, the results in [38] only involve open-loop control design using an idea of changing controller structures and do not consider the robustness which can be obtained through sliding mode control. Ref. [38] and Ref. [40] have briefly discussed the possible application of sliding mode control to quantum systems. In [41], two approaches based on sliding mode design have been proposed for the control of quantum systems and potential applications of sliding mode control to quantum information processing have been presented. Following these results, this paper formally presents a sliding mode control method for two-level quantum systems to deal with bounded uncertainties in the system Hamiltonian [42]. In particular, we propose two approaches of designing the measurement period for different situations which are dependent on the bound on the uncertainties and the allowed probability of failure. 
Variable structure control design with sliding modes generally includes two main steps: selecting a sliding surface (sliding mode) and controlling the system to and maintaining it in this sliding surface. Being in the sliding surface guarantees that the quantum system has the desired dynamics. We will select an eigenstate of the free Hamiltonian of the controlled quantum system as a sliding mode. In the second step, direct feedback control is not directly applicable since we generally cannot acquire state information without destroying the quantum system's state. Hence, we propose a new method to accomplish this task, which is based on the Lyapunov methodology and periodic projective measurements. The Lyapunov methodology is a powerful tool for designing control laws in classical control theory and has also been applied to quantum control problems [43]-[48]. Most existing results on Lyapunov control of quantum systems focus on designing a control law to ensure that the controlled quantum system's state asymptotically converges to the target state. The existing Lyapunov design methods in quantum control rely on perfect knowledge of the initial quantum states and system Hamiltonian. In our approach, once the Lyapunov control steers the quantum system into a sliding mode domain, we make a projective measurement on the system. Hence, the Lyapunov design method can tolerate small drifts (uncertainties) when carrying out our control tasks, which will be demonstrated by simulation in Section II.C. Periodic projective measurements are employed to maintain the system's state in the sliding mode domain when uncertainties exist in the system Hamiltonian. If the measurement period is small enough and the initial state is an eigenstate, the frequent measurements make the system collapse back to the initial state. This is related to the quantum Zeno effect (for details, see [49], [50] and [51]). In contrast to the quantum Zeno effect, our objective is to design a measurement period which is as large as possible. The framework of the proposed method involves unitary control (Lyapunov control) and projective measurement. In this sense, it is similar to the discrete-time quantum feedback stabilization problem in [52] and [53]. However, these papers do not consider possible uncertainties in the system Hamiltonian and use generalized measurements rather than periodic projective measurements. The main feature of the proposed method is that the control law can guarantee control performance when bounded uncertainties exist in the system Hamiltonian.

This paper is organized as follows. Section $\amalg$ introduces a quantum control model, defines the sliding mode and formulates the control problem. In Section III, we present a sliding mode control method based on the Lyapunov methodology and periodic projective measurements for two-level 
quantum systems with bounded uncertainties. Using the known information about uncertainties (e.g., the uncertainty bound and type of uncertainties), we propose two approaches (i.e., Eqs. (13) and (14)) for designing the measurement period to guarantee the control performance. An illustrative example is presented to demonstrate the proposed method. The detailed proofs of the main theorems are presented in Section IV. Conclusions are given in Section $\mathrm{V}$

\section{SLIDING MODES AND PROBLEM FORMULATION}

In this section, we first introduce a two-level quantum control model. Then a sliding mode is defined using an eigenstate. Finally the control problem considered in this paper is formulated.

\section{A. Quantum Control Model}

In this paper, we focus on two-level pure-state quantum systems. The quantum state can be represented by a two-dimensional unit vector $|\psi\rangle$ in a Hilbert space $\mathscr{H}$. Since the global phase of a quantum state has no observable physical effect, we do not consider the effect of global phase. If we denote the Pauli matrices $\sigma=\left(\sigma_{x}, \sigma_{y}, \sigma_{z}\right)$ as follows:

$$
\sigma_{x}=\left(\begin{array}{cc}
0 & 1 \\
1 & 0
\end{array}\right), \quad \sigma_{y}=\left(\begin{array}{cc}
0 & -i \\
i & 0
\end{array}\right), \quad \sigma_{z}=\left(\begin{array}{cc}
1 & 0 \\
0 & -1
\end{array}\right),
$$

we may select the free Hamiltonian of the two-level quantum system as $H_{0}=I_{z}=\frac{1}{2} \sigma_{z}$. Its two eigenstates are denoted as $|0\rangle$ and $|1\rangle$. To control a quantum system, we introduce the following control Hamiltonian $H_{u}=\sum_{k} u_{k}(t) H_{k}$, where $u_{k}(t) \in \mathbf{R}$ and $\left\{H_{k}\right\}$ is a set of time-independent Hamiltonians. For simplicity, the control Hamiltonian for two-level systems can be written as $H_{u}=u_{x}(t) I_{x}+u_{y}(t) I_{y}+u_{z}(t) I_{z}$, where

$$
I_{x}=\frac{1}{2} \sigma_{x}=\frac{1}{2}\left(\begin{array}{ll}
0 & 1 \\
1 & 0
\end{array}\right), \quad I_{y}=\frac{1}{2} \sigma_{y}=\frac{1}{2}\left(\begin{array}{cc}
0 & -i \\
i & 0
\end{array}\right) .
$$

The controlled dynamical equation can be described as (we have assumed $\hbar=1$ by using atomic units in this paper)

$$
\begin{aligned}
& i|\dot{\psi}(t)\rangle=H_{0}|\psi(t)\rangle+\sum_{k=x, y, z} u_{k}(t) I_{k}|\psi(t)\rangle, \\
& |\psi(t=0)\rangle=\left|\psi_{0}\right\rangle .
\end{aligned}
$$


This control problem is converted into the following problem: given an initial state and a target state, find a set of controls $\left\{u_{k}(t)\right\}$ in (3) to drive the controlled system from the initial state to the target state.

In practical applications, we often use the density operator (or density matrix) $\rho$ to describe the quantum state of a quantum system. For a pure state $|\psi\rangle$, the corresponding density operator is $\rho \equiv|\psi\rangle\langle\psi|$. For a two-level quantum system, the state $\rho$ can be represented in terms of the Bloch vector $\mathbf{r}=(x, y, z)=\left(\operatorname{tr}\left\{\rho \sigma_{x}\right\}, \operatorname{tr}\left\{\rho \sigma_{y}\right\}, \operatorname{tr}\left\{\rho \sigma_{z}\right\}\right)$ :

$$
\rho=\frac{1}{2}(I+\mathbf{r} \cdot \sigma)
$$

The evolution equation of $\rho$ can be written as

$$
\dot{\rho}=-i[H, \rho]
$$

where $[A, B]=A B-B A$ and $H$ is the total system Hamiltonian.

After we represent the state $\rho$ with the Bloch vector, the pure states of a two-level quantum system correspond to the surface of the Bloch sphere, where $(x, y, z)=(\sin \theta \cos \varphi, \sin \theta \sin \varphi, \cos \theta)$, $\theta \in[0, \pi], \varphi \in[0,2 \pi]$. An arbitrary pure state $|\psi\rangle$ for a two-level quantum system can be represented as

$$
|\psi\rangle=\cos \frac{\theta}{2}|0\rangle+e^{i \varphi} \sin \frac{\theta}{2}|1\rangle
$$

\section{B. Sliding Modes}

Sliding modes play an important role in variable structure control [36]. Usually, the sliding mode is constructed so that the system has desired dynamics in the sliding surface. For a quantum control problem, a sliding mode may be represented as a functional of the state $|\psi\rangle$ and the Hamiltonian $H$; i.e., $S(|\psi\rangle, H)=0$. For example, an eigenstate $\left|\phi_{j}\right\rangle$ of the free Hamiltonian $H_{0}$ (i.e., $H_{0}\left|\phi_{j}\right\rangle=\lambda_{j}\left|\phi_{j}\right\rangle$ where $\lambda_{j}$ is one eigenvalue of $H_{0}$ ) can be selected as a sliding mode. We can define $S(|\psi\rangle, H)=1-\left|\left\langle\psi \mid \phi_{j}\right\rangle\right|^{2}=0$. If the initial state $\left|\psi_{0}\right\rangle$ is in the sliding mode; i.e., $S\left(\left|\psi_{0}\right\rangle, H\right)=1-\left|\left\langle\psi_{0} \mid \phi_{j}\right\rangle\right|^{2}=0$, we can easily prove that the quantum system will maintain its state in this surface under only the action of the free Hamiltonian $H_{0}$. In fact, $|\psi(t)\rangle=e^{-i H_{0} t}\left|\psi_{0}\right\rangle$, and we have

$$
\begin{aligned}
S(\mid \psi(t), H) & =1-\left|\left\langle\psi(t) \mid \phi_{j}\right\rangle\right|^{2}=1-\left|\left\langle\psi_{0}\left|e^{i H_{0} t}\right| \phi_{j}\right\rangle\right|^{2} \\
& =1-\left|\left\langle\psi_{0} \mid \phi_{j}\right\rangle e^{i \lambda_{j} t}\right|^{2}=1-\left|\left\langle\psi_{0} \mid \phi_{j}\right\rangle\right|^{2}\left|e^{i \lambda_{j} t}\right|^{2} \\
& =0 .
\end{aligned}
$$


That is, an eigenstate of $H_{0}$ can be identified as a sliding mode. For two-level quantum systems, we may select either $|0\rangle$ or $|1\rangle$ as a sliding mode. Without loss of generality, we identify the eigenstate $|0\rangle$ of a two-level quantum system as the sliding mode in this paper.

\section{Problem Formulation}

In Section II.B, we have identified an eigenstate $|0\rangle$ as a sliding mode. This means that if a quantum system is driven into the sliding mode, its state will be maintained in the sliding surface under the action of the free Hamiltonian. However, in practical applications, it is inevitable that there exist noises and uncertainties. In this paper, we suppose that the uncertainties can be approximately described as perturbations in the Hamiltonian. That is, the uncertainties can be denoted as $H_{\Delta}=\varepsilon_{x}(t) I_{x}+\varepsilon_{y}(t) I_{y}+\varepsilon_{z}(t) I_{z}$. The unitary errors in [33] belong to this class of uncertainties and uncertainties in one-qubit (one quantum bit) gate also correspond to this class of uncertainties [41]. For a spin system in solid-state nuclear magnetic resonance (NMR), external noisy magnetic fields and unwanted coupling with other spins may lead to uncertainties in this class. Further, we assume the uncertainties are bounded; i.e.,

$$
\sqrt{\varepsilon_{x}^{2}(t)+\varepsilon_{y}^{2}(t)+\varepsilon_{z}^{2}(t)} \leq \varepsilon \quad(\varepsilon \geq 0) .
$$

When $\varepsilon=0, H_{\Delta}=0$. That is, there exist no uncertainties, which is trivial for our problem. Hence, in the following we assume $\varepsilon>0$. An important advantage of classical sliding mode control is its robustness. Our main motivation for introducing sliding mode control to quantum systems is to deal with uncertainties. We further suppose that the corresponding system without uncertainties is completely controllable and arbitrary unitary control operations can be generated. This assumption can be guaranteed for a two-level quantum system if we can realize arbitrary rotations along the $z$-axis and $\zeta$-axis ( $\zeta=x$ or $y$ ) (e.g., see [54] for details).

The control problem under consideration is stated as follows: design a control law to drive and then maintain the quantum system's state in a sliding mode domain even when bounded uncertainties exist in the system Hamiltonian. Here a sliding mode domain may be defined as $\mathscr{D}=\left\{|\psi\rangle:|\langle 0 \mid \psi\rangle|^{2} \geq 1-p_{0}, 0<p_{0}<1\right\}$, where $p_{0}$ is a given constant. Here we assume $p_{0} \neq 0,1$ since the case $p_{0}=0$ only occurs in the sliding mode surface and the case $p_{0}=1$ is always true. Hence, the two cases with $p_{0}=0$ and $p_{0}=1$ are trivial for our problem. The definition of the sliding mode domain implies that the system has a probability of at most $p_{0}$ (which we call 
the probability of failure) to collapse out of $\mathscr{D}$ when making a measurement. This behavior is quite different from that which occurs in traditional sliding mode control. Hence, we expect that our control laws will guarantee that the system's state remains in $\mathscr{D}$ except that a measurement operation may take it away from $\mathscr{D}$ with a small probability (not greater than $p_{0}$ ). The control problem considered in this paper includes three main subtasks: (i) for any initial state (assumed to be known), design a control law to drive the system's state into a defined sliding mode domain $\mathscr{D}$; (ii) design a control law to maintain the system's state in $\mathscr{D}$; (iii) design a control law to drive the system's state back to $\mathscr{D}$ if a measurement operation takes it away from $\mathscr{D}$. For simplicity, we suppose that there exist no uncertainties during the control processes (i) and (iii).

\section{SLiding mode CONTROL BASEd ON LyAPUnOV METHOdS AND PROJECTIVE MEASUREMENTS}

\section{A. General Method}

The first task is to design a control law to drive the controlled system to the chosen sliding mode domain $\mathscr{D}$. Lyapunov-based methods are widely used to accomplish this task in traditional sliding mode control. If the gradient of a Lyapunov function is negative in the neighborhood of the sliding surface, then the controlled system's state will be attracted to and maintained in $\mathscr{D}$. The Lyapunov methodology has also been used to design control laws for quantum systems [43][48]. However, these existing results do not consider the issue of robustness against uncertainties. Since the measurement of a quantum system will inevitably destroy the measured state, most existing results on Lyapunov-based control for quantum systems in fact use a feedback design to construct an open-loop control. That is, Lyapunov-based control can be used to first design a feedback law which is then used to find the open-loop control by simulating the closed-loop system. Then the control can be applied to the quantum system in an open-loop way. Hence, the traditional sliding mode control methods using Lyapunov control cannot be directly applied to our problem.

Although quantum measurement often has deleterious effects in quantum control tasks, recent results have shown that it can be combined with unitary transformations to complete some quantum manipulation tasks and enhance the capability of quantum control [40], [55]-[59]. For example, Vilela Mendes and Man'ko [40] showed nonunitarily controllable systems can be made controllable by using "measurement plus evolution". Quantum measurement can be used as a 
control tool as well as a method of information acquisition. It is worth mentioning that the effect of measurement on a quantum system as a control tool can be achieved through the interaction between the system and measurement apparatus. In this paper, we will combine the Lyapunov methodology and projective measurements (with the measurement operator $\sigma_{z}$ ) to accomplish the sliding mode control task for two-level quantum systems. The projective measurement with $\sigma_{z}$ on a two-level system makes the system's state collapse into $|0\rangle$ (corresponding to eigenvalue 1 of $\sigma_{z}$ ) or $|1\rangle$ (corresponding to eigenvalue -1 of $\sigma_{z}$ ).

The steps in the control algorithm are as follows (see Fig. 1):

1) Select an eigenstate $|0\rangle$ of $H_{0}$ as a sliding mode $S(|\psi\rangle, H)=0$, and define the sliding mode domain as $\mathscr{D}=\left\{|\psi\rangle:|\langle 0 \mid \psi\rangle|^{2} \geq 1-p_{0}\right\}$.

2) For a known initial state $\left|\psi_{0}\right\rangle$, construct a Lyapunov function $V\left(\left|\psi_{0}\right\rangle, S\right)$ to find the control law that can drive $\left|\psi_{0}\right\rangle$ into the sliding mode $S$.

3) For a specified probability of failure $p_{0}$ and $V\left(\left|\psi_{0}\right\rangle, S\right)$, construct the control period $T_{0}$ so that the control law can drive the system's state into $\mathscr{D}$ in a time period $T_{0}$.

4) For an initial condition which is another eigenstate $|1\rangle$, design a Lyapunov function $V(|1\rangle, S)$ and construct the period $T_{1}$ by using a similar method to that in 3$)$.

5) According to $p_{0}$ and $\varepsilon$, design the period $T$ for periodic projective measurements.

6) Use the designed control law to drive the system's state into $\mathscr{D}$ in $T_{0}$ and make a projective measurement at $t=T_{0}$. Then repeat the following operations: make periodic projective measurements with the period $T$ to maintain the system's state in $\mathscr{D}$; if the measurement result corresponds to $|1\rangle$, we use the corresponding control law to drive the state back into $\mathscr{D}$.

From the above control algorithm, we see that the design of Lyapunov functions and the selection of the period $T$ for the projective measurements are the two most important tasks. To design a control law for quantum systems, several Lyapunov functions have been constructed, such as state distance-based and average value-based approaches [43]-[48]. Here we select a function based on the Hilbert-Schmidt distance between a state $|\psi\rangle$ and the sliding mode state $\left|\phi_{j}\right\rangle$ as a Lyapunov function [46], [48]; i.e.,

$$
V(|\psi\rangle, S)=\frac{1}{2}\left(1-\left|\left\langle\phi_{j} \mid \psi\right\rangle\right|^{2}\right) .
$$



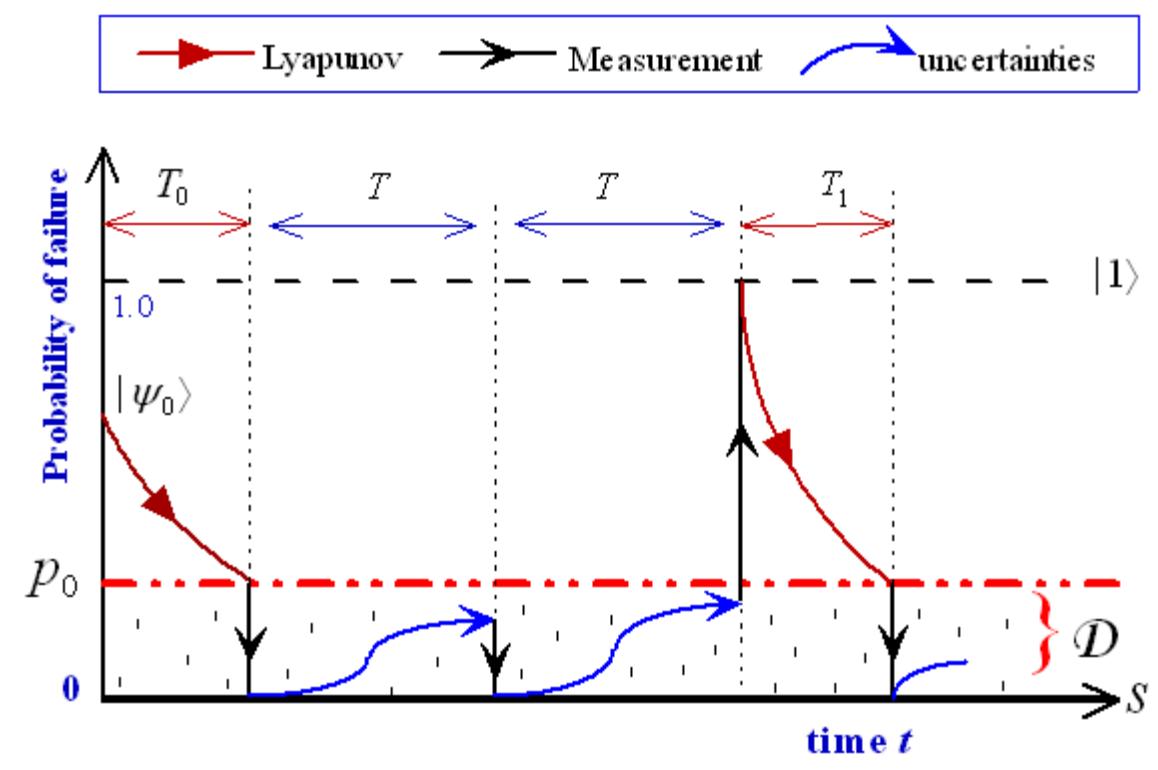

Fig. 1. The sliding mode control scheme for a two-level quantum system based on Lyapunov methods and periodic projective measurements. In this figure, "Lyapunov", "Measurement" and "uncertainties" mean the evolution process of the quantum system under the Lyapunov control law, the projective measurement and uncertainties in the system Hamiltonian, respectively.

It is clear that $V \geq 0$. The first-order time derivative of $V$ is

$$
\begin{aligned}
\dot{V} & =-\sum_{k=x, y, z} u_{k} \mathfrak{I}\left[\left\langle\psi \mid \phi_{j}\right\rangle\left\langle\phi_{j}\left|I_{k}\right| \psi\right\rangle\right] \\
& =-\sum_{k=x, y, z} u_{k}\left|\left\langle\psi \mid \phi_{j}\right\rangle\right| \mathfrak{I}\left[e^{i \angle\left\langle\psi \mid \phi_{j}\right\rangle}\left\langle\phi_{j}\left|I_{k}\right| \psi\right\rangle\right],
\end{aligned}
$$

where $\mathfrak{I}[a+b i]=b(a, b \in \mathbf{R})$ and $\angle c$ denotes the argument of a complex number $c$. To ensure $\dot{V} \leq 0$, we choose the control laws as in [48]:

$$
u_{k}=K_{k} f_{k}\left(\mathfrak{I}\left[e^{i<\left\langle\psi \mid \phi_{j}\right\rangle}\left\langle\phi_{j}\left|I_{k}\right| \psi\right\rangle\right]\right), \quad(k=x, y, z)
$$

where $K_{k}>0$ may be used to adjust the control amplitude and $f(\cdot)$ satisfies $x f(x) \geq 0$. Define $\angle\left\langle\psi \mid \phi_{j}\right\rangle=0^{\circ}$ when $\left\langle\psi \mid \phi_{j}\right\rangle=0$.

When one employs a Lyapunov methodology to design a control law, LaSalle's invariance principle is a useful tool to analyze its convergence. That is, if $\dot{x}(t)=g(x(t))$ is an autonomous dynamical system with phase space $\Omega$ and $V(x)$ is a Lyapunov function on $\Omega$ satisfying $V(x)>$ 0 for all $x \neq x_{0}$ and $\dot{V}(x(t)) \leq 0$, any bounded solution converges to the invariant set $E=$ $\{x \mid \dot{V}(x(t))=0\}$ as $t \rightarrow+\infty$ (for details, see [60]). For two-level quantum systems, LaSalle's invariance principle can guarantee that the quantum state converges to the sliding mode $|0\rangle$ under the control law in (10) (for details, see [48]). The convergence is asymptotic. Hence, we 
make a projective measurement with the measurement operator $\sigma_{z}$ when we apply the Lyapunov control to the system for $T_{0}$ (corresponding to the initial condition $\left|\psi_{0}\right\rangle$ ) or $T_{1}$ (corresponding to the initial condition $|1\rangle$ ), which will drive the system into $|0\rangle$ with a probability not less than $1-p_{0}$.

Another important task is to design the measurement period $T$. We can estimate a bound according to the bound $\varepsilon$ on the uncertainties and the allowed probability of failure $p_{0}$. Then, we construct a period $T$ to guarantee control performance according to the estimated bound. An extreme case is $T \rightarrow 0$. That is, after the quantum system's state is driven into the sliding mode, we make frequent measurements. This corresponds to the quantum Zeno effect [50], which is the inhibition of transitions between quantum states by frequent measurement of the state (see, e.g., [49] and [50]). Frequent measurements (i.e., $T \rightarrow 0$ ) can guarantee that the state is maintained in the sliding mode in spite of the presence of uncertainties. However, it is usually a difficult task to make such frequent measurements. We may conclude that the smaller $T$ is, the bigger the cost of accomplishing the periodic measurements becomes. Hence, in contrast to the quantum Zeno effect, we wish to design a measurement period $T$ as large as possible. In the following subsection, we will propose two approaches of designing $T$ for different situations.

\section{B. The Design of the Measurement Period T}

We select the sliding mode as $S(|\psi\rangle, H)=1-|\langle\psi \mid 0\rangle|^{2}=0$. If there exist no uncertainties and we have driven the system's state to the sliding mode at time $t_{0}$, it will be maintained in the sliding mode using only the free Hamiltonian $H_{0}$; i.e., $S\left(\left|\psi_{\left(t \geq t_{0}\right)}\right\rangle, H_{0}\right)=0$. That is, if the quantum system's state is driven into the sliding mode, it will evolve in the sliding surface. However, in practical applications, some uncertainties are unavoidable, which may drive the system's state away from the sliding mode. We wish to design a control law to ensure the desired robustness in the presence of uncertainties. Assume that the state at time $t$ is $\rho_{t}$. If we make measurements on this system, the probability $p$ that it will collapse into $|1\rangle$ (the probability of failure) is

$$
p=\left\langle 1\left|\rho_{t}\right| 1\right\rangle=\frac{1-z_{t}}{2}
$$

where $z_{t}=\operatorname{tr}\left(\rho_{t} \sigma_{z}\right)$. We have assumed that the possible uncertainties can be described by $H_{\Delta}=$ $\varepsilon_{x}(t) I_{x}+\varepsilon_{y}(t) I_{y}+\varepsilon_{z}(t) I_{z}$, where unknown $\varepsilon_{x}(t), \varepsilon_{y}(t)$ and $\varepsilon_{z}(t)$ satisfy $\sqrt{\varepsilon_{x}^{2}(t)+\varepsilon_{y}^{2}(t)+\varepsilon_{z}^{2}(t)} \leq \varepsilon$. We now give detailed discussions to design the measurement period $T$ for possible uncertainties. 
First we consider a special case $H_{\Delta}=\varepsilon(t) I_{z}(|\varepsilon(t)| \leq \varepsilon)$. This case corresponds to phase-flip type bounded uncertainties. For any $H_{\Delta}=\varepsilon_{z} I_{z}$ (where $\left|\varepsilon_{z}\right| \leq \varepsilon$ ), if $S\left(\left|\psi_{0}\right\rangle, H\right)=0$, we have

$$
\begin{aligned}
S(|\psi(t)\rangle, H) & =1-|\langle\psi(t) \mid 0\rangle|^{2} \\
& =1-\left|\left\langle\psi_{0}\left|e^{i\left(H_{0}+\varepsilon_{z} I_{z}\right) t}\right| 0\right\rangle\right|^{2} \\
& =1-\left|\left\langle\psi_{0} \mid 0\right\rangle\right|^{2}\left|e^{\frac{i\left(1+\varepsilon_{z}\right)}{2} t}\right|^{2} \\
& =0 .
\end{aligned}
$$

This type of uncertainty does not drive the system's state away from the sliding mode. Hence we ignore this type of uncertainty in our method.

Now we consider the unknown uncertainties $H_{\Delta}=\varepsilon_{x}(t) I_{x}+\varepsilon_{y}(t) I_{y}\left(\right.$ where $\left.\sqrt{\varepsilon_{x}^{2}(t)+\varepsilon_{y}^{2}(t)} \leq \varepsilon\right)$ and have the following theorem.

Theorem 1: For a two-level quantum system with the initial state $|\psi(0)\rangle=|0\rangle$ at the time $t=0$, the system evolves to $|\psi(t)\rangle$ under the action of $H(t)=I_{z}+\varepsilon_{x}(t) I_{x}+\varepsilon_{y}(t) I_{y}$ (where $\sqrt{\varepsilon_{x}^{2}(t)+\varepsilon_{y}^{2}(t)} \leq \varepsilon$ and $\left.\varepsilon>0\right)$. If $t \in\left[0, T^{(1)}\right]$, where

$$
T^{(1)}=\frac{\arccos \left(1-2 p_{0}\right)}{\varepsilon}
$$

the system's state will remain in $\mathscr{D}=\left\{|\psi\rangle:|\langle 0 \mid \psi\rangle|^{2} \geq 1-p_{0}\right\}$ (where $0<p_{0}<1$ ). When one makes a projective measurement with the measurement operator $\sigma_{z}$ at the time $t$, the probability of failure $p=|\langle 1 \mid \psi(t)\rangle|^{2}$ is not greater than $p_{0}$.

Using Theorem 1, we may try to maintain the system's state in $\mathscr{D}$ (i.e., the subtask (ii)) by implementing periodic projective measurements with the measurement period $T=T^{(1)}$. If we have more knowledge about the uncertainties, it is possible to improve the measurement period $T^{(1)}$. Now assume that the uncertainty is $H_{\Delta}=\varepsilon(t) I_{\zeta}(\zeta=x$ or $y)$ and $p_{0} \in\left(0, \frac{\varepsilon^{2}}{1+\varepsilon^{2}}\right]$. We have the following theorem.

Theorem 2: For a two-level quantum system with the initial state $|\psi(0)\rangle=|0\rangle$ at the time $t=0$, the system evolves to $|\psi(t)\rangle$ under the action of $H(t)=I_{z}+\varepsilon(t) I_{\zeta}$ (where $\zeta=x$ or $y$, $|\varepsilon(t)| \leq \varepsilon$ and $\varepsilon>0)$. If $p_{0} \in\left(0, \frac{\varepsilon^{2}}{1+\varepsilon^{2}}\right]$ and $t \in\left[0, T^{(2)}\right]$, where

$$
T^{(2)}=\frac{\arccos \left[1-2\left(1+\frac{1}{\varepsilon^{2}}\right) p_{0}\right]}{\sqrt{1+\varepsilon^{2}}},
$$

the system's state will remain in $\mathscr{D}=\left\{|\psi\rangle:|\langle 0 \mid \psi\rangle|^{2} \geq 1-p_{0}\right\}$ (where $0<p_{0}<1$ ). When one makes a projective measurement with the measurement operator $\sigma_{z}$ at the time $t$, the probability of failure $p=|\langle 1 \mid \psi(t)\rangle|^{2}$ is not greater than $p_{0}$. 
Remark 1: The proofs of Theorem 1 and Theorem 2 will be presented in Section IV. The two theorems mean the following fact. For a two-level quantum system with unknown uncertainties $H_{\Delta}=\varepsilon_{x}(t) I_{x}+\varepsilon_{y}(t) I_{y}$ (where $\sqrt{\varepsilon_{x}^{2}(t)+\varepsilon_{y}^{2}(t)} \leq \varepsilon$ ), if its initial state is in the sliding mode $|0\rangle$, we can ensure that the probability of failure is not greater than a given constant $p_{0}\left(0<p_{0}<1\right)$ through implementing periodic projective measurements with the measurement period $T=T^{(1)}$ using (13). Further, if we know that $p_{0}$ and $\varepsilon$ satisfy the relationship $0<$ $p_{0} \leq \frac{\varepsilon^{2}}{1+\varepsilon^{2}}$ and there exists only one type of uncertainty (i.e., $H_{\Delta}=\varepsilon(t) I_{x}$ or $H_{\Delta}=\varepsilon(t) I_{y}$, where $|\varepsilon(t)| \leq \varepsilon$ ), we can design a measurement period $T=T^{(2)}$ using (14) which is larger than $T^{(1)}$. The proof of Theorem 2 also shows that $T^{(2)}$ is an optimal measurement period. This measurement period will guarantee the required robustness. It is easy to prove the relationship $T^{(2)} \geq T^{(1)}$ for arbitrary $p_{0} \in\left(0, \frac{\varepsilon^{2}}{1+\varepsilon^{2}}\right]$. The detailed proof will be presented in the Appendix. Based on the above analysis, the selection rule for $T$ is summarized in Table I. Moreover, from (13) and (14), it is clear that for a constant $\varepsilon, T^{(1)} \rightarrow 0$ and $T^{(2)} \rightarrow 0$ when $p_{0} \rightarrow 0$. That is, for a given bound $\varepsilon$ on the uncertainties, if we expect to guarantee the probability of failure $p_{0} \rightarrow 0$, it requires us to implement frequent measurements such that the measurement period $T \rightarrow 0$. Another special case is $\varepsilon \rightarrow+\infty$, which leads to $T^{(1)} \rightarrow 0$ and $T^{(2)} \rightarrow 0$. That is, to deal with very large uncertainties, we need to make frequent measurements $(T \rightarrow 0)$ to guarantee the desired robustness. From (13), we also know that for a given $p_{0}, T^{(1)}$ monotonically decreases with increasing $\varepsilon$. This means that we need to employ a smaller measurement period to deal with uncertainties with a larger bound $\varepsilon$.

\begin{tabular}{|c|c|c|c|}
\hline Type of uncertainties & $H_{\Delta}=\varepsilon_{x}(t) I_{x}+\varepsilon_{y}(t) I_{y}$ & \multicolumn{2}{|c|}{$H_{\Delta}=\varepsilon(t) I_{\zeta}(\zeta=x$ or $y)$} \\
\hline Allowed probability of failure $p_{0}$ & $0<p_{0}<1$ & $0<p_{0} \leq \frac{\varepsilon^{2}}{1+\varepsilon^{2}}$ & $\frac{\varepsilon^{2}}{1+\varepsilon^{2}}<p_{0}<1$ \\
\hline The measurement period $T$ & $T=T^{(1)}$ & $T=T^{(2)}$ & $T=T^{(1)}$ \\
\hline
\end{tabular}

TABLE I

A SUMMARY ON THE SELECTION RULE OF THE MEASUREMENT PERIOD $T$

\section{An Illustrative Example}

Now we present an illustrative example to demonstrate the proposed method. Assume $p_{0}=$ 0.01. Consider two cases: (a) $\varepsilon=0.02$; (b) $\varepsilon=0.2$. For simplicity, we assume $\left|\psi_{0}\right\rangle=|1\rangle$. 


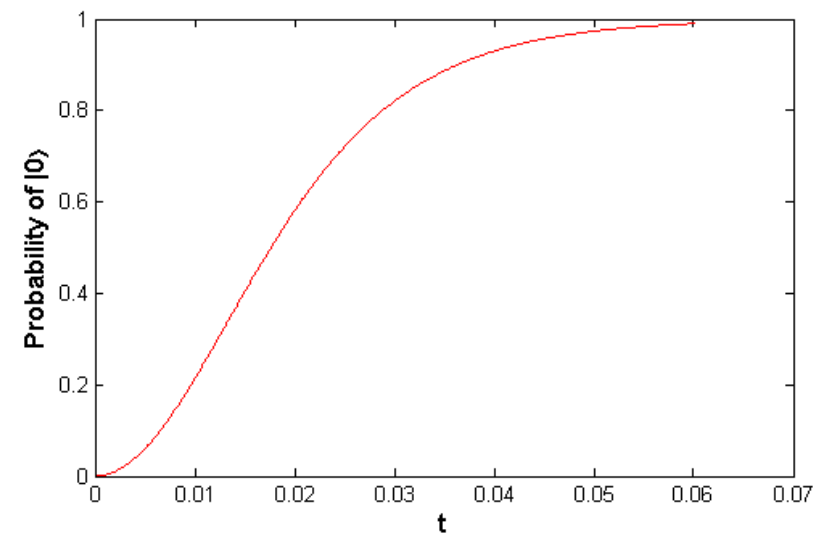

Fig. 2. The probability of $|0\rangle$ under Lyapunov control.

Hence, $T_{0}=T_{1}$. We first design the control and $T_{1}$ using (10). Here, we consider control only using $H_{u}=\frac{1}{2} u(t) \sigma_{y}$. Using (10), we select $u(t)=K\left(\mathfrak{I}\left[e^{i \angle\langle\psi(t) \mid 0\rangle}\left\langle 0\left|\sigma_{y}\right| \psi(t)\right\rangle\right]\right)$ and $K=100$. Let the time stepsize be given by $\delta t=10^{-4}$. We can obtain the probability curve of $|0\rangle$ shown in Fig. 2, the control value shown in Fig. 3 and $T_{1}=0.060$. For $\varepsilon=0.02$, we can design the measurement period $T=T^{(1)}=10.017$ using (13). For $\varepsilon=0.2$, we can design the measurement period $T=T^{(1)}=1.002$ using (13). Since $p^{\prime}=\frac{\varepsilon^{2}}{1+\varepsilon^{2}}=3.8 \times 10^{-2}>p_{0}$ when $\varepsilon=0.2$, if the uncertainties take the form of $H_{\Delta}=\varepsilon(t) I_{\zeta}(\zeta=x$ or $y)$, we can improve the measurement period to $T=T^{(2)}=1.049$ using (14). It is clear that $T \gg T_{1}$ in these two cases. For some practical quantum systems such as spin systems in NMR, we can use strong control actions (e.g., $K=10^{5}$ ) to drive the system from $|1\rangle$ into $\mathscr{D}$ within a short time period $T_{1}$ [8]. These facts make the assumption of no uncertainties in the control process reasonable. Moreover, the fact that the measurement period $T$ is much greater than the control time required to go to $|0\rangle$ from $|1\rangle$ indicates the possibility of realizing such a periodic measurement on a practical quantum system.

Remark 2: In the process of designing the control law for driving the system's state from $|1\rangle$ to $|0\rangle$, we employ an approach based on the Lyapunov methodology. An advantage of such an approach is that it is relatively easy to find a control law by simulation. It is worth noting that most existing applications of the Lyapunov methodology to quantum systems do not involve measurement. Here, we combine the Lyapunov-based control and projective measurements for 


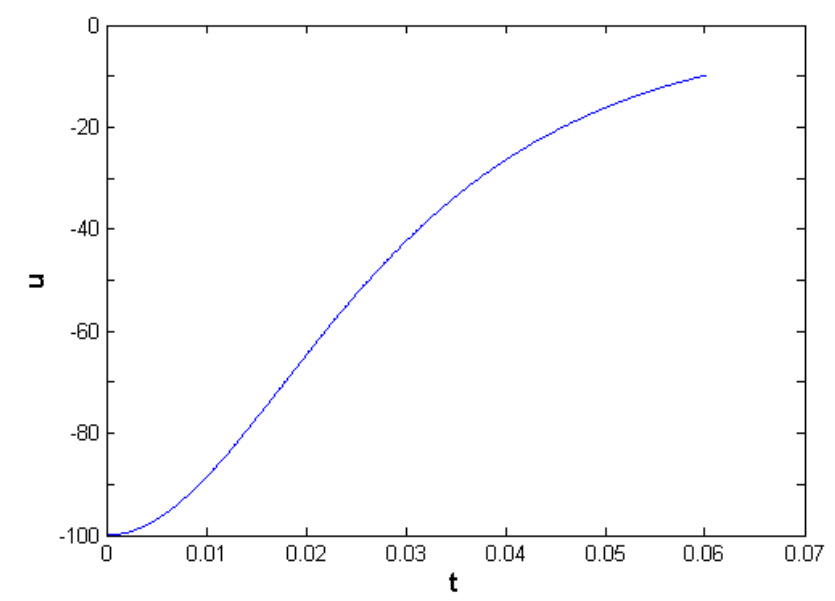

Fig. 3. The control value $u(t)$.

controlling quantum systems, which in some applications make our method more useful than the Lyapunov-based control for quantum systems proposed in previous papers. In [41], an approach based on time-optimal control design has also been proposed to complete this task. The advantage of such an approach is that we take the shortest time to complete the control task. However, it is generally difficult to find a complete time-optimal solution for high-dimensional quantum systems. For the above simple task, it has been proven that the time-optimal control employs a bang-bang control strategy [14]. Using the method in [14], we should take $u=-100$ in $t \in[0,0.016]$ and then use $u=100$ in $t \in(0.016,0.030]$. In this case, the total time required is $T_{1}^{\prime}=0.030\left(<T_{1}=0.060\right)$.

Remark 3: In the process of designing the Lyapunov control for driving the system's state from $|1\rangle$ to $|0\rangle$, we ignore possible uncertainties. By simulation, we find that small uncertainties can also be tolerated in this process. For example, if $\varepsilon=0.02$ and the uncertainty $\varepsilon(t)$ is the noise with a uniform distribution on the interval $[-0.02,0.02]$, the probability curves of $|0\rangle$ are shown in Fig. 4 when we apply the control obtained from Fig. 3 to the quantum system. The probabilities of $|0\rangle$ for the cases with uncertainties are very close to the probability of $|0\rangle$ for the case without uncertainties. By more simulation, we find that the final probability of $|0\rangle$ is $(99.00 \pm 0.01) \%$ for $\varepsilon(t) I_{x}(|\varepsilon(t)| \leq \varepsilon$ where $\varepsilon=0.02$ or 0.2$)$, the final probability of $|0\rangle$ is $(99.00 \pm 0.02) \%$ for $\varepsilon(t) I_{y}(|\varepsilon(t)| \leq 0.02)$ and the final probability of $|0\rangle$ is $(99.00 \pm 0.13) \%$ for $\varepsilon(t) I_{y}(|\varepsilon(t)| \leq 0.2)$. If we use a smaller probability of failure $\tilde{p}_{0}$ (e.g., $\left.\tilde{p}_{0}=0.5 p_{0}\right)$ as 


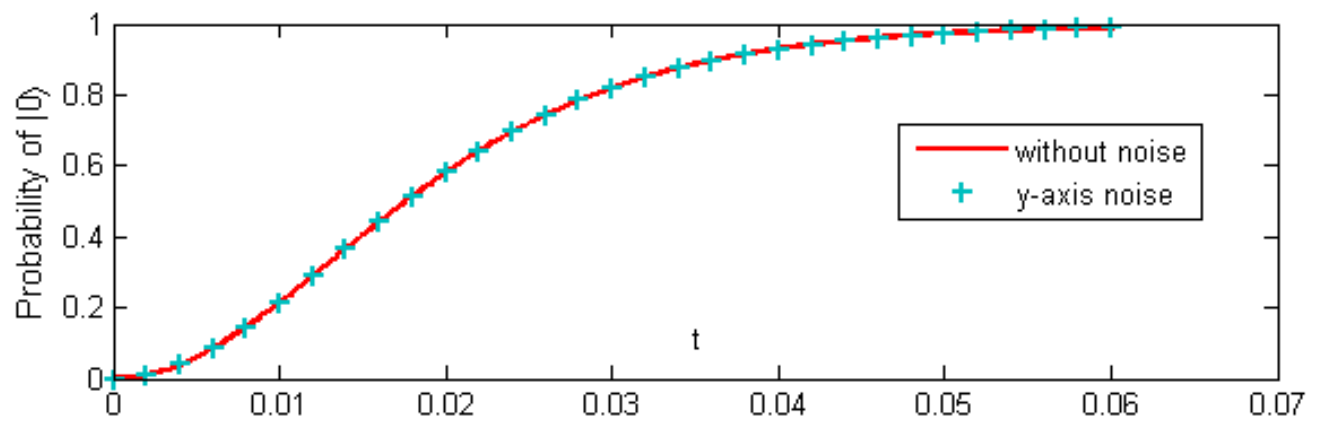

(a) Comparison between the case without noise and the case with $y$-axis noise

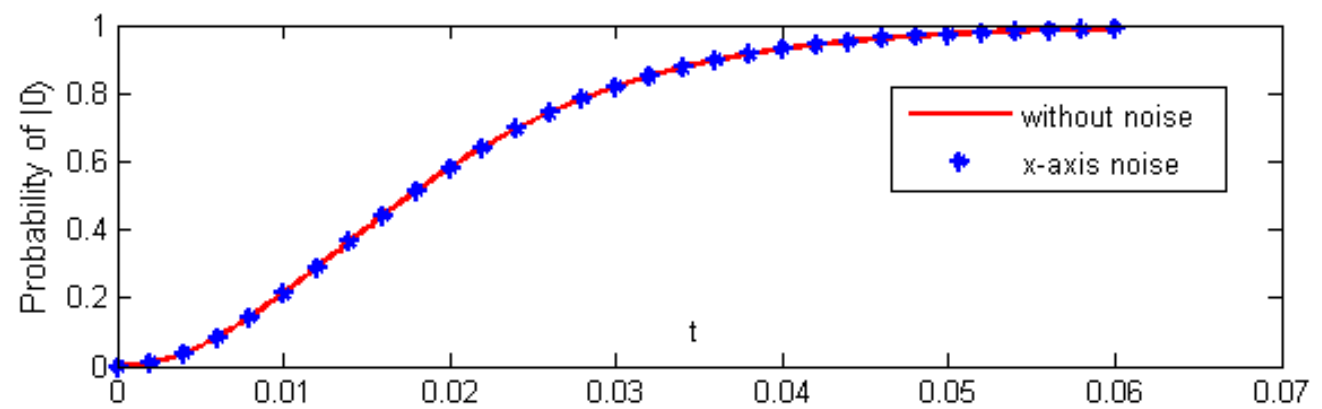

(b) Comparison between the case without noise and the case with $x$-axis noise

Fig. 4. The probability curves of $|0\rangle$ for the case without uncertainties (without noise) and the cases with uncertainties (noise) when we apply the control in Fig. 3 to the quantum system. The $\xi$-axis noise $\left(\xi=x\right.$ or $y$ ) means the existence of $\varepsilon(t) I_{\xi}$ where $\varepsilon(t)$ is the noise with a uniform distribution on the interval $[-0.02,0.02]$.

the terminal condition of the Lyapunov control or employ a bigger $K$ for the same $T_{1}$, these simulations suggest that it is possible to ensure that the Lyapunov control will drive the system's state into the sliding mode domain even when there exist small uncertainties.

\section{Proof of Theorems}

This section will present the detailed proofs of Theorem 1 and Theorem 2. The proof of Theorem 1 involves the following steps: (I) Compare the probabilities of failure for $H=I_{z}+$ $\varepsilon_{0} \cos \gamma_{0} I_{x}+\varepsilon_{0} \sin \gamma_{0} I_{y}$ and $H=\varepsilon_{0} \cos \gamma_{0} I_{x}+\varepsilon_{0} \sin \gamma_{0} I_{y}\left(\varepsilon_{0}\right.$ and $\gamma_{0}$ are constant); (II) Compare the probabilities of failure for $H=\varepsilon I_{x}$ and $H=I_{z}+\varepsilon(t) I_{x}(|\varepsilon(t)| \leq \varepsilon)$; (III) Use the previous results to compare the probabilities of failure for $H=\varepsilon I_{x}$ and $H=I_{z}+\varepsilon_{x}(t) I_{x}+\varepsilon_{y}(t) I_{y}\left(\sqrt{\varepsilon_{x}^{2}(t)+\varepsilon_{y}^{2}(t)} \leq\right.$ $\varepsilon)$; (IV) Use $H=\varepsilon I_{x}$ to estimate the measurement period. The basic steps for the proof of Theorem 2 include: (I) Formulate the problem of finding the "worst" case as an optimal control 
problem of min $z_{f}$; (II) Obtain the optimal control solution for nonsingular cases; (III) Exclude the possibility of singular cases; (IV) Use the "worst" case to estimate the measurement period. Considering that the arguments in the proof of Theorem 2 are useful for the proof of Theorem 1, we will first present the proof of Theorem 2 and then prove Theorem 1.

\section{A. Proof of Theorem 2}

Proof: For $H^{A}=I_{z}+\varepsilon(t) I_{x}$, using $\dot{\rho}=-i\left[H^{A}, \rho\right]$ and (4), we have

$$
\left(\begin{array}{cc}
\dot{z}_{t} & \dot{x}_{t}-i \dot{y}_{t} \\
\dot{x}_{t}+i \dot{y}_{t} & -\dot{z}_{t}
\end{array}\right)=\left(\begin{array}{cc}
\varepsilon(t) y_{t} & -y_{t}-i x_{t}+i \varepsilon(t) z_{t} \\
-y_{t}+i x_{t}-i \varepsilon(t) z_{t} & -\varepsilon(t) y_{t}
\end{array}\right) .
$$

That is,

$$
\left(\begin{array}{c}
\dot{x}_{t} \\
\dot{y}_{t} \\
\dot{z}_{t}
\end{array}\right)=\left(\begin{array}{ccc}
0 & -1 & 0 \\
1 & 0 & -\varepsilon(t) \\
0 & \varepsilon(t) & 0
\end{array}\right)\left(\begin{array}{c}
x_{t} \\
y_{t} \\
z_{t}
\end{array}\right)
$$

where $\left(x_{0}, y_{0}, z_{0}\right)=(0,0,1)$.

We now consider $\varepsilon(t)$ as a control input and select the performance measure as

$$
J(\varepsilon)=z_{f} .
$$

From (11), we know that the "worst" case (i.e., the case maximizing the probability of failure) corresponds to minimizing $z_{f}$. Also, we introduce the Lagrange multiplier vector $\lambda(t)=$ $\left(\lambda_{1}(t), \lambda_{2}(t), \lambda_{3}(t)\right)^{T}$ and obtain the corresponding Hamiltonian function as follows:

$$
\mathbb{H}(\mathbf{r}(t), \varepsilon(t), \lambda(t), t) \equiv \lambda^{T}(t)\left(\begin{array}{ccc}
0 & -1 & 0 \\
1 & 0 & -\varepsilon(t) \\
0 & \varepsilon(t) & 0
\end{array}\right)\left(\begin{array}{c}
x_{t} \\
y_{t} \\
z_{t}
\end{array}\right),
$$

where $\mathbf{r}(t)=\left(x_{t}, y_{t}, z_{t}\right)$. That is

$$
\mathbb{H}(\mathbf{r}(t), \varepsilon(t), \lambda(t), t)=-\lambda_{1}(t) y_{t}+\lambda_{2}(t) x_{t}+\varepsilon(t)\left(\lambda_{3}(t) y_{t}-\lambda_{2}(t) z_{t}\right)
$$

According to Pontryagin's minimum principle [61], a necessary condition for $\varepsilon^{*}(t)$ to minimize $J(\varepsilon)$ is

$$
\mathbb{H}\left(\mathbf{r}^{*}(t), \varepsilon^{*}(t), \lambda^{*}(t), t\right) \leq \mathbb{H}\left(\mathbf{r}^{*}(t), \varepsilon(t), \lambda^{*}(t), t\right) .
$$

The necessary condition provides a relationship to determine the optimal control $\varepsilon^{*}(t)$. If there exists a time interval $\left[t_{1}, t_{2}\right]$ of finite duration during which the necessary condition (20) provides 
no information about the relationship between $\mathbf{r}^{*}(t), \varepsilon^{*}(t), \lambda^{*}(t)$, we call the interval $\left[t_{1}, t_{2}\right]$ a singular interval [61]. If we do not consider singular cases (i.e., $\lambda_{3}(t) y_{t}-\lambda_{2}(t) z_{t} \equiv 0$ ), the optimal control $\varepsilon^{*}(t)$ should be chosen as follows:

$$
\varepsilon^{*}(t)=-\varepsilon \operatorname{sgn}\left(\lambda_{3}(t) y_{t}-\lambda_{2}(t) z_{t}\right)
$$

That is, the optimal control strategy for $\varepsilon(t)$ is bang-bang control; i.e., $\varepsilon^{*}(t)=\bar{\varepsilon}=+\varepsilon$ or $-\varepsilon$. Now we consider $H^{B}=I_{z}+\bar{\varepsilon} I_{x}$ which leads to the state equation

$$
\left(\begin{array}{c}
\dot{x}_{t} \\
\dot{y}_{t} \\
\dot{z}_{t}
\end{array}\right)=\left(\begin{array}{ccc}
0 & -1 & 0 \\
1 & 0 & -\bar{\varepsilon} \\
0 & \bar{\varepsilon} & 0
\end{array}\right)\left(\begin{array}{l}
x_{t} \\
y_{t} \\
z_{t}
\end{array}\right)
$$

where $\left(x_{0}, y_{0}, z_{0}\right)=(0,0,1)$. The corresponding solution is

$$
\left(\begin{array}{l}
x_{t} \\
y_{t} \\
z_{t}
\end{array}\right)=\left(\begin{array}{c}
-\frac{\bar{\varepsilon}}{1+\varepsilon^{2}} \cos \omega t+\frac{\bar{\varepsilon}}{1+\varepsilon^{2}} \\
-\frac{\bar{\varepsilon}}{\sqrt{1+\varepsilon^{2}}} \sin \omega t \\
\frac{\varepsilon^{2}}{1+\varepsilon^{2}} \cos \omega t+\frac{1}{1+\varepsilon^{2}}
\end{array}\right)
$$

where $\omega=\sqrt{1+\varepsilon^{2}}$. From (23), we know that $z_{t}$ is a monotonically decreasing function in $t$ when $t \in\left[0, \frac{\pi}{\sqrt{1+\varepsilon^{2}}}\right]$. Hence, we only consider the case $t \in\left[0, t_{f}\right]$ where $t_{f} \in\left[0, \frac{\pi}{\sqrt{1+\varepsilon^{2}}}\right]$.

Now consider the optimal control problem with a fixed final time $t_{f}$ and a free final state $\mathbf{r}_{f}=\left(x_{f}, y_{f}, z_{f}\right)$. According to Pontryagin's minimum principle, $\lambda^{*}\left(t_{f}\right)=\frac{\partial}{\partial \mathbf{r}} \mathbf{r}^{*}\left(t_{f}\right)$. From this, it is straightforward to verify that $\left(\lambda_{1}\left(t_{f}\right), \lambda_{2}\left(t_{f}\right), \lambda_{3}\left(t_{f}\right)\right)=(0,0,1)$. Now let us consider another necessary condition $\dot{\lambda}(t)=-\frac{\partial \mathbb{H}(\mathbf{r}(t), \varepsilon(t), \lambda(t), t)}{\partial \mathbf{r}}$ which leads to the following relationships:

$$
\dot{\lambda}(t)=\left(\begin{array}{c}
\dot{\lambda}_{1}(t) \\
\dot{\lambda}_{2}(t) \\
\dot{\lambda}_{3}(t)
\end{array}\right)=\left(\begin{array}{ccc}
0 & -1 & 0 \\
1 & 0 & -\bar{\varepsilon} \\
0 & \bar{\varepsilon} & 0
\end{array}\right)\left(\begin{array}{c}
\lambda_{1}(t) \\
\lambda_{2}(t) \\
\lambda_{3}(t)
\end{array}\right),
$$

where $\left(\lambda_{1}\left(t_{f}\right), \lambda_{2}\left(t_{f}\right), \lambda_{3}\left(t_{f}\right)\right)=(0,0,1)$. The corresponding solution is

$$
\left(\begin{array}{l}
\lambda_{1}(t) \\
\lambda_{2}(t) \\
\lambda_{3}(t)
\end{array}\right)=\left(\begin{array}{c}
-\frac{\bar{\varepsilon}}{1+\varepsilon^{2}} \cos \omega\left(t_{f}-t\right)+\frac{\bar{\varepsilon}}{1+\varepsilon^{2}} \\
\frac{\bar{\varepsilon}}{\sqrt{1+\varepsilon^{2}}} \sin \omega\left(t_{f}-t\right) \\
\frac{\varepsilon^{2}}{1+\varepsilon^{2}} \cos \omega\left(t_{f}-t\right)+\frac{1}{1+\varepsilon^{2}}
\end{array}\right) .
$$


We obtain

$$
\lambda_{3}(t) y_{t}-\lambda_{2}(t) z_{t}=\frac{-\bar{\varepsilon}}{\omega^{3}}\left[\sin \omega t+\varepsilon^{2} \sin \omega t_{f}+\sin \omega\left(t_{f}-t\right)\right] .
$$

It is easy to show that the quantity $\left(\lambda_{3}(t) y_{t}-\lambda_{2}(t) z_{t}\right)$ occurring in (21) does not change its sign when $t_{f} \in\left[0, \frac{\pi}{\sqrt{1+\varepsilon^{2}}}\right]$ and $t \in\left[0, t_{f}\right]$.

Now we further exclude the possibility that there exists a singular case. Suppose that there exists a singular interval $\left[t_{0}, t_{1}\right]\left(\right.$ where $\left.t_{0} \geq 0\right)$ such that when $t \in\left[t_{0}, t_{1}\right]$

$$
h(t)=\lambda_{3}(t) y_{t}-\lambda_{2}(t) z_{t} \equiv 0 .
$$

We also have the following relationship

$$
\dot{h}(t)=\lambda_{3}(t) x_{t}-\lambda_{1}(t) z_{t} \equiv 0
$$

where we have used (16) and the following costate equation

$$
\dot{\lambda}(t)=\left(\begin{array}{c}
\dot{\lambda}_{1}(t) \\
\dot{\lambda}_{2}(t) \\
\dot{\lambda}_{3}(t)
\end{array}\right)=\left(\begin{array}{ccc}
0 & -1 & 0 \\
1 & 0 & -\varepsilon(t) \\
0 & \varepsilon(t) & 0
\end{array}\right)\left(\begin{array}{c}
\lambda_{1}(t) \\
\lambda_{2}(t) \\
\lambda_{3}(t)
\end{array}\right) .
$$

If $t_{0}=0$, we have $\left(x_{0}, y_{0}, z_{0}\right)=(0,0,1)$. By the principle of optimality [61], we may consider the case $t_{f}=t_{1}$. Using (27), (28) and $\left(\lambda_{1}\left(t_{1}\right), \lambda_{2}\left(t_{1}\right), \lambda_{3}\left(t_{1}\right)\right)=(0,0,1)$, we have $x_{t_{1}}=0$ and $y_{t_{1}}=0$. Using the relationship of $x_{t}^{2}+y_{t}^{2}+z_{t}^{2}=1$, we obtain $z_{t_{1}}=1$ or -1 . If $z_{t_{1}}=1$, the initial state and the final state are the same state $|0\rangle$. However, if we use the control $\varepsilon(t)=\bar{\varepsilon}$, from (23) we have $z_{t_{1}}(\bar{\varepsilon})=\frac{\varepsilon^{2}}{1+\varepsilon^{2}} \cos \omega t_{1}+\frac{1}{1+\varepsilon^{2}}<z_{t_{1}}=1$. Hence, this contradicts the fact that we are considering the optimal case $\min z_{f}$. If $z_{t_{1}}=-1$, there exists $0<\tilde{t}_{1}<t_{1}$ such that $z_{\tilde{t}_{1}}=0$. By the principle of optimality [61], we may consider the case $t_{f}=\tilde{t}_{1}$. From the two equations (27) and (28), we know that $z_{\tilde{t}_{1}}^{2}=1$ which contradicts $z_{\tilde{t}_{1}}=0$. Hence, no singular condition can exist if $t_{0}=0$.

If $t_{0}>0$, using (21) we must select $\varepsilon(t)=\bar{\varepsilon}$ when $t \in\left[0, t_{0}\right]$. From (26), we know that there exist no $t_{0} \in\left(0, t_{f}\right)$ satisfying $\lambda_{3}\left(t_{0}\right) y_{t_{0}}-\lambda_{2}\left(t_{0}\right) z_{t_{0}}=0$. Hence, there exist no singular cases for our problem.

From the above analysis, $\varepsilon(t)=\bar{\varepsilon}$ is the optimal control when $t \in\left[0, \frac{\pi}{\sqrt{1+\varepsilon^{2}}}\right]$. Hence $z_{t}^{A}=$ $z_{t}(\varepsilon(t)) \geq z_{t}(\bar{\varepsilon})=z_{t}^{B}$. From (11), it is clear that the probabilities of failure satisfy $p_{t}^{A}=\frac{1-z_{t}^{A}}{2} \leq$ $p_{t}^{B}=\frac{1-z_{t}^{B}}{2}$. That is, the probability of failure $p_{t}^{A}$ is not greater than $p_{t}^{B}$ for $t \in\left[0, \frac{\pi}{\sqrt{1+\varepsilon^{2}}}\right]$. When 
$t \in\left[0, \frac{\pi}{\sqrt{1+\varepsilon^{2}}}\right], z_{t}^{B}$ is monotonically decreasing and $p_{t}^{B}$ is monotonically increasing. When $t=$ $\frac{\pi}{\sqrt{1+\varepsilon^{2}}}$, using (23) we have $z_{t}^{B}=\frac{1-\varepsilon^{2}}{1+\varepsilon^{2}}$. That is, the probability of failure $p^{\prime}=\frac{\varepsilon^{2}}{1+\varepsilon^{2}}$. Hence, we can design the measurement period $T$ using the case of $H^{B}$ when $0<p_{0} \leq \frac{\varepsilon^{2}}{1+\varepsilon^{2}}$.

Using (11) and (23), for $t \in\left[0, \frac{\pi}{\sqrt{1+\varepsilon^{2}}}\right]$ we obtain the probability of failure

$$
p_{t}^{B}=\frac{\varepsilon^{2}}{1+\varepsilon^{2}} \frac{1-\cos \omega t}{2} .
$$

Hence, we can design the maximum measurement period as follows

$$
T^{(2)}=\frac{\arccos \left[1-2\left(1+\frac{1}{\varepsilon^{2}}\right) p_{0}\right]}{\sqrt{1+\varepsilon^{2}}},
$$

For $H_{\Delta}=\varepsilon(t) I_{y}$ (where $|\varepsilon(t)| \leq \varepsilon$ ), we can obtain the same conclusion as that in the case $H_{\Delta}=\varepsilon(t) I_{x}($ where $|\varepsilon(t)| \leq \varepsilon)$.

\section{B. Proof of Theorem 1}

To prove Theorem 1, we first prove two lemmas (Lemma 3 and Lemma 5).

Lemma 3: For a two-level quantum system with the initial state $\left(x_{0}, y_{0}, z_{0}\right)=(0,0,1)$ (i.e., $|0\rangle)$, the system evolves to $\left(x_{t}^{A}, y_{t}^{A}, z_{t}^{A}\right)$ and $\left(x_{t}^{B}, y_{t}^{B}, z_{t}^{B}\right)$ under the action of $H^{A}=I_{z}+\varepsilon_{0} \cos \gamma_{0} I_{x}+$ $\varepsilon_{0} \sin \gamma_{0} I_{y}$ ( $\varepsilon_{0}$ is a nonzero constant) and $H^{B}=\varepsilon_{0} \cos \gamma_{0} I_{x}+\varepsilon_{0} \sin \gamma_{0} I_{y}$, respectively. For arbitrary $t \in\left[0, \frac{\pi}{\left|\varepsilon_{0}\right|}\right], z_{t}^{A} \geq z_{t}^{B}$.

Proof: For the system with Hamiltonian $H^{A}=I_{z}+\varepsilon_{0} \cos \gamma_{0} I_{x}+\varepsilon_{0} \sin \gamma_{0} I_{y}$, using $\dot{\rho}=-i[H, \rho]$ and (4), we obtain the following state equations

$$
\left(\begin{array}{c}
\dot{x}_{t}^{A} \\
\dot{y}_{t}^{A} \\
\dot{z}_{t}^{A}
\end{array}\right)=\left(\begin{array}{ccc}
0 & -1 & \varepsilon_{0} \sin \gamma_{0} \\
1 & 0 & -\varepsilon_{0} \cos \gamma_{0} \\
-\varepsilon_{0} \sin \gamma_{0} & \varepsilon_{0} \cos \gamma_{0} & 0
\end{array}\right)\left(\begin{array}{c}
x_{t}^{A} \\
y_{t}^{A} \\
z_{t}^{A}
\end{array}\right) \text {, }
$$

where $\left(x_{0}^{A}, y_{0}^{A}, z_{0}^{A}\right)=(0,0,1)$. The corresponding solution is as follows

$$
\left(\begin{array}{c}
x_{t}^{A} \\
y_{t}^{A} \\
z_{t}^{A}
\end{array}\right)=\left(\begin{array}{c}
\frac{\varepsilon_{0} \sin \gamma_{0}}{\sqrt{1+\varepsilon_{0}^{2}}} \sin \omega_{0} t-\frac{\varepsilon_{0} \cos \gamma_{0}}{1+\varepsilon_{0}^{2}} \cos \omega_{0} t+\frac{\varepsilon_{0} \cos \gamma_{0}}{1+\varepsilon_{0}^{2}} \\
-\frac{\varepsilon_{0} \cos \gamma_{0}}{\sqrt{1+\varepsilon_{0}^{2}}} \sin \omega_{0} t-\frac{\varepsilon_{0} \sin \gamma_{0}}{1+\varepsilon_{0}^{2}} \cos \omega_{0} t+\frac{\varepsilon_{0} \sin \gamma_{0}}{1+\varepsilon_{0}^{2}} \\
\frac{\varepsilon_{0}^{2}}{1+\varepsilon_{0}^{2}} \cos \omega_{0} t+\frac{1}{1+\varepsilon_{0}^{2}}
\end{array}\right) .
$$

where $\omega_{0}=\sqrt{1+\varepsilon_{0}^{2}}$. 
For the system with Hamiltonian $H^{B}=\varepsilon_{0} \cos \gamma_{0} I_{x}+\varepsilon_{0} \sin \gamma_{0} I_{y}$, using $\dot{\rho}=-i[H, \rho]$ and (4), we obtain the following state equations

$$
\left(\begin{array}{c}
\dot{x}_{t}^{B} \\
\dot{y}_{t}^{B} \\
\dot{z}_{t}^{B}
\end{array}\right)=\left(\begin{array}{ccc}
0 & 0 & \varepsilon_{0} \sin \gamma_{0} \\
0 & 0 & -\varepsilon_{0} \cos \gamma_{0} \\
-\varepsilon_{0} \sin \gamma_{0} & \varepsilon_{0} \cos \gamma_{0} & 0
\end{array}\right)\left(\begin{array}{c}
x_{t}^{B} \\
y_{t}^{B} \\
z_{t}^{B}
\end{array}\right),
$$

where $\left(x_{0}^{B}, y_{0}^{B}, z_{0}^{B}\right)=(0,0,1)$. We can obtain the corresponding solution as

$$
\left(\begin{array}{c}
x_{t}^{B} \\
y_{t}^{B} \\
z_{t}^{B}
\end{array}\right)=\left(\begin{array}{c}
\sin \gamma_{0} \sin \varepsilon_{0} t \\
-\cos \gamma_{0} \sin \varepsilon_{0} t \\
\cos \varepsilon_{0} t
\end{array}\right)
$$

Since $\cos \varepsilon_{0} t=\cos \left(-\varepsilon_{0} t\right)$, we may first assume $\varepsilon_{0}>0$. We define $F(t)$ and $f(t)$ as follows.

$$
\begin{gathered}
F(t)=z_{t}^{A}-z_{t}^{B}, \\
f(t)=F^{\prime}(t)=\varepsilon_{0} \sin \varepsilon_{0} t-\frac{\varepsilon_{0}^{2}}{\sqrt{1+\varepsilon_{0}^{2}}} \sin \omega_{0} t .
\end{gathered}
$$

Now we consider $t \in\left[0, \frac{\pi}{\sqrt{1+\varepsilon_{0}^{2}}}\right]$, and obtain

$$
f^{\prime}(t)=\varepsilon_{0}^{2}\left(\cos \varepsilon_{0} t-\cos \omega_{0} t\right)=2 \varepsilon_{0}^{2} \sin \frac{\omega_{0}+\varepsilon_{0}}{2} t \sin \frac{\omega_{0}-\varepsilon_{0}}{2} t \geq 0
$$

It is clear that $f^{\prime}(t)=0$ only when $t=0$. Hence $f(t)$ is a monotonically increasing function and

$$
\min _{t} f(t)=f(0)=0
$$

Hence, we have

$$
f(t) \geq 0
$$

From this, it is clear that $F(t)$ is a monotonically increasing function and

$$
\min _{t} F(t)=F(0)=0
$$

Hence $F(t) \geq 0$ when $t \in\left[0, \frac{\pi}{\sqrt{1+\varepsilon_{0}^{2}}}\right]$. Moreover, it is clear that $\min _{t} z_{t}^{A}=\left.z_{t}^{A}\right|_{t=\frac{\pi}{\sqrt{1+\varepsilon_{0}^{2}}}}$ and $z_{t}^{B}=\cos \varepsilon_{0} t$ is a monotonically decreasing function when $t \in\left[0, \frac{\pi}{\left|\varepsilon_{0}\right|}\right]$. It is easy to obtain the following relationship

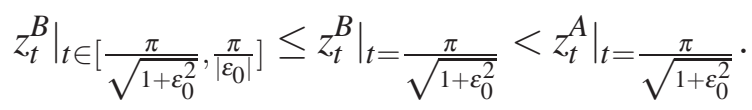


Hence we can conclude that $z_{t}^{A} \geq z_{t}^{B}$ for arbitrary $t \in\left[0, \frac{\pi}{\left|\varepsilon_{0}\right|}\right]$.

Let $\gamma_{0}=0$, and we have the following corollary.

Corollary 4: For a two-level quantum system with the initial state $\left(x_{0}, y_{0}, z_{0}\right)=(0,0,1)$ (i.e., $|0\rangle)$, the system evolves to $\left(x_{t}^{A}, y_{t}^{A}, z_{t}^{A}\right)$ and $\left(x_{t}^{B}, y_{t}^{B}, z_{t}^{B}\right)$ under the action of $H^{A}=I_{z}+\varepsilon_{0} I_{x}$ (where $\varepsilon_{0}$ is a nonzero constant) and $H^{B}=\varepsilon_{0} I_{x}$, respectively. For arbitrary $t \in\left[0, \frac{\pi}{\left|\varepsilon_{0}\right|}\right], z_{t}^{A} \geq z_{t}^{B}$.

We now present another lemma.

Lemma 5: For a two-level quantum system with the initial state $\left(x_{0}, y_{0}, z_{0}\right)=(0,0,1)$ (i.e., $|0\rangle$ ), the system evolves to $\left(x_{t}^{A}, y_{t}^{A}, z_{t}^{A}\right)$ and $\left(x_{t}^{B}, y_{t}^{B}, z_{t}^{B}\right)$ under the action of $H^{A}=I_{z}+\varepsilon(t) I_{x}$ (where $|\varepsilon(t)| \leq \varepsilon)$ and $H^{B}=\varepsilon I_{x}$, respectively. For arbitrary $t \in\left[0, \frac{\pi}{\varepsilon}\right], z_{t}^{A} \geq z_{t}^{B}$.

Proof: First, we take an arbitrary evolution state (except $|1\rangle$ ) starting from $|0\rangle$ as a new initial state. For $H^{B}=\varepsilon I_{x}$, the initial state can be represented as $\left(x_{0}^{\prime}, y_{0}^{\prime}, z_{0}\right)=\left(0,-\sin \theta_{0}, \cos \theta_{0}\right)$, where $\theta_{0} \in[0, \pi)$. We have

$$
\left(\begin{array}{c}
\dot{x}_{t}^{B} \\
\dot{y}_{t}^{B} \\
\dot{z}_{t}^{B}
\end{array}\right)=\left(\begin{array}{ccc}
0 & 0 & 0 \\
0 & 0 & -\varepsilon \\
0 & \varepsilon & 0
\end{array}\right)\left(\begin{array}{c}
x_{t}^{B} \\
y_{t}^{B} \\
z_{t}^{B}
\end{array}\right) .
$$

The corresponding solution is as follows:

$$
\left(\begin{array}{c}
x_{t}^{B} \\
y_{t}^{B} \\
z_{t}^{B}
\end{array}\right)=\left(\begin{array}{c}
0 \\
-z_{0} \sin \varepsilon t+y_{0}^{\prime} \cos \varepsilon t \\
z_{0} \cos \varepsilon t+y_{0}^{\prime} \sin \varepsilon t
\end{array}\right) .
$$

For $H^{A}=I_{z}+\varepsilon(t) I_{x}$, we have

$$
\left(\begin{array}{c}
\dot{x}_{t}^{A} \\
\dot{y}_{t}^{A} \\
\dot{z}_{t}^{A}
\end{array}\right)=\left(\begin{array}{ccc}
0 & -1 & 0 \\
1 & 0 & -\varepsilon(t) \\
0 & \varepsilon(t) & 0
\end{array}\right)\left(\begin{array}{c}
x_{t}^{A} \\
y_{t}^{A} \\
z_{t}^{A}
\end{array}\right),
$$

where $\left(x_{0}^{A}, y_{0}^{A}, z_{0}^{A}\right)=\left(x_{0}, y_{0}, z_{0}\right)=\left(\sin \theta_{0} \cos \varphi_{0}, \sin \theta_{0} \sin \varphi_{0}, \cos \theta_{0}\right)$ and $\varphi_{0} \in[0,2 \pi]$. From (42), we have

$$
\left.\dot{z}_{t}\right|_{t=0}=\lim _{\Delta t \rightarrow 0} \frac{z_{\Delta t}^{A}-z_{0}}{\Delta t}=\varepsilon(0) y_{0}=\varepsilon(0) \sin \theta_{0} \sin \varphi_{0} .
$$

From (43) and (41), it is easy to obtain the following relationship:

$$
\begin{aligned}
z_{\Delta t}^{A}-z_{\Delta t}^{B} & =z_{0}+\varepsilon(0) \sin \theta_{0} \sin \varphi_{0} \Delta t-z_{0}\left[1-\frac{\varepsilon^{2}(\Delta t)^{2}}{2}\right]+\sin \theta_{0} \varepsilon \Delta t+O\left((\Delta t)^{2}\right) \\
& =\Delta t \sin \theta_{0}\left(\varepsilon+\varepsilon(0) \sin \varphi_{0}\right)+O\left((\Delta t)^{2}\right) .
\end{aligned}
$$


When $\theta_{0} \in[0, \pi), \sin \theta_{0} \geq 0$. Moreover, it is always true that $\varepsilon+\varepsilon(0) \sin \varphi_{0} \geq 0$. If $\theta_{0} \neq 0$ and $\varepsilon+\varepsilon(0) \sin \varphi_{0} \neq 0$, we have

$$
z_{\Delta t}^{A}>z_{\Delta t}^{B}
$$

If $\theta_{0}=0$, we have $\left(x_{0}^{\prime}, y_{0}^{\prime}, z_{0}\right)=\left(x_{0}, y_{0}, z_{0}\right)=(0,0,1)$. According to Corollary 4 and the proof of Theorem 2, we have

$$
z_{\Delta t}^{A} \geq z_{\Delta t}^{B}
$$

For $\varepsilon+\varepsilon(0) \sin \varphi_{0}=0$, it corresponds to two cases: (a) $\varepsilon(0)=\varepsilon$ and $\varphi_{0}=\frac{3 \pi}{2}$; (b) $\varepsilon(0)=-\varepsilon$ and $\varphi_{0}=\frac{\pi}{2}$. In the following, we consider the case (a) (for the case (b) we have the same conclusion as the case (a)). Since $\varphi_{0}=\frac{3 \pi}{2},\left(x_{0}, y_{0}, z_{0}\right)=\left(0,-\sin \theta_{0}, \cos \theta_{0}\right)$. Using a similar argument to the proof of Theorem 2, we know that for $H^{A}=I_{z}+\varepsilon(t) I_{z}$ with $\left(x_{0}, y_{0}, z_{0}\right)=$ $\left(0,-\sin \theta_{0}, \cos \theta_{0}\right)$, the optimal control for the performance index $J(\varepsilon)=z_{f}$ takes a form of bang-bang control $\varepsilon(t)=\bar{\varepsilon}=\varepsilon$ or $-\varepsilon$. So we only need to consider a bang-bang strategy.

For such a bang-bang strategy as $H^{A}=I_{z}+\bar{\varepsilon} I_{x}$, we have

$$
\left(\begin{array}{c}
\dot{x}_{t}^{A} \\
\dot{y}_{t}^{A} \\
\dot{z}_{t}^{A}
\end{array}\right)=\left(\begin{array}{ccc}
0 & -1 & 0 \\
1 & 0 & -\bar{\varepsilon} \\
0 & \bar{\varepsilon} & 0
\end{array}\right)\left(\begin{array}{c}
x_{t}^{A} \\
y_{t}^{A} \\
z_{t}^{A}
\end{array}\right)
$$

where $\left(x_{0}^{A}, y_{0}^{A}, z_{0}^{A}\right)=\left(x_{0}, y_{0}, z_{0}\right)=\left(0,-\sin \theta_{0}, \cos \theta_{0}\right)$ and $\varphi_{0} \in[0,2 \pi]$. We can obtain the corresponding solution as

$$
\left(\begin{array}{c}
x_{t}^{A} \\
y_{t}^{A} \\
z_{t}^{A}
\end{array}\right)=\left(\begin{array}{c}
\frac{-\bar{\varepsilon} \cos \theta_{0}}{1+\varepsilon^{2}} \cos \omega t+\frac{\sin \theta_{0}}{\sqrt{1+\varepsilon^{2}}} \sin \omega t+\frac{\bar{\varepsilon} \cos \theta_{0}}{1+\varepsilon^{2}} \\
\frac{-\bar{\varepsilon} \cos \theta_{0}}{\sqrt{1+\varepsilon^{2}}} \sin \omega t-\sin \theta_{0} \cos \omega t \\
\frac{\varepsilon^{2} \cos \theta_{0}}{1+\varepsilon^{2}} \cos \omega t-\frac{\bar{\varepsilon} \sin \theta_{0}}{\sqrt{1+\varepsilon^{2}}} \sin \omega t+\frac{\cos \theta_{0}}{1+\varepsilon^{2}}
\end{array}\right) .
$$

Now, we consider the limit as $\Delta t \rightarrow 0$ and obtain

$$
\begin{aligned}
z_{\Delta t}^{A}-z_{\Delta t}^{B}= & \frac{\varepsilon^{2} \cos \theta_{0}}{1+\varepsilon^{2}}\left[1-\frac{\left(1+\varepsilon^{2}\right)(\Delta t)^{2}}{2}\right]-\frac{\bar{\varepsilon} \sin \theta_{0}}{\sqrt{1+\varepsilon^{2}}} \sqrt{1+\varepsilon^{2}} \Delta t \\
& +\frac{\cos \theta_{0}}{1+\varepsilon^{2}}-\cos \theta_{0}\left[1-\frac{\varepsilon^{2}(\Delta t)^{2}}{2}\right]+\sin \theta_{0} \varepsilon \Delta t-\frac{\varepsilon^{3}}{6}(\Delta t)^{3} \sin \theta_{0}+O\left((\Delta t)^{4}\right) \\
= & \Delta t \sin \theta_{0}(\varepsilon-\bar{\varepsilon})+\sin \theta_{0}(\Delta t)^{3}\left(\bar{\varepsilon}+\bar{\varepsilon} \varepsilon^{2}-\varepsilon^{3}\right)+O\left((\Delta t)^{4}\right) \\
> & 0
\end{aligned}
$$

Hence, for arbitrary $z_{0}=\cos \theta_{0}\left(\theta_{0} \in[0, \pi)\right)$, we have

$$
z_{\Delta t}^{A} \geq z_{\Delta t}^{B}
$$


For $t=\frac{\pi}{\varepsilon}, z_{t}^{B}=-1$. Hence the relationship $z_{t}^{A} \geq z_{t}^{B}$ is always true.

We now define $g(t)=z_{t}^{A}-z_{t}^{B}$ and assume that there exist $t=t_{1} \in\left[0, \frac{\pi}{\varepsilon}\right)$ such that $z_{t_{1}}^{A}<z_{t_{1}}^{B}$. That is, $g\left(t_{1}\right)<0$. Since $g(t)$ is continuous in $t$ and $g(0)=0$, there exists a time $t^{*}=\sup \{t \mid 0 \leq t<$ $\left.t_{1}, g(t)=0\right\}$ satisfying $g(t)<0$ for $t \in\left(t^{*}, t_{1}\right]$. However, we have proven that for any $z_{t}^{A}=z_{t}^{B}$ and $\Delta t \rightarrow 0, z_{t+\Delta t}^{A} \geq z_{t+\Delta t}^{B}$, which contradicts $g(t)<0$ for $t \in\left(t^{*}, t_{1}\right]$. Hence, we have the following relationship for $t \in\left[0, \frac{\pi}{\varepsilon}\right]$

$$
z_{t}^{A} \geq z_{t}^{B}
$$

Now we can prove Theorem 1 using Lemma 3 and Lemma 5 ,

Proof: For $H^{A}=I_{z}+\varepsilon_{x}(t) I_{x}+\varepsilon_{y}(t) I_{y}$, using $\dot{\rho}=-i[H, \rho]$ and (4), we can obtain the following state equations

$$
\left(\begin{array}{c}
\dot{x}_{t}^{A} \\
\dot{y}_{t}^{A} \\
\dot{z}_{t}^{A}
\end{array}\right)=\left(\begin{array}{ccc}
0 & -1 & \varepsilon_{y}(t) \\
1 & 0 & -\varepsilon_{x}(t) \\
-\varepsilon_{y}(t) & \varepsilon_{x}(t) & 0
\end{array}\right)\left(\begin{array}{c}
x_{t}^{A} \\
y_{t}^{A} \\
z_{t}^{A}
\end{array}\right)
$$

where $\left(x_{0}^{A}, y_{0}^{A}, z_{0}^{A}\right)=(0,0,1)$.

Define $\varepsilon(t)=\sqrt{\varepsilon_{x}^{2}(t)+\varepsilon_{y}^{2}(t)}$ and $\varepsilon_{x}(t)=\varepsilon(t) \cos \gamma_{t}, \varepsilon_{y}(t)=\varepsilon(t) \sin \gamma_{t}$. This leads to the following equation

$$
\left(\begin{array}{c}
\dot{x}_{t}^{A} \\
\dot{y}_{t}^{A} \\
\dot{z}_{t}^{A}
\end{array}\right)=\left(\begin{array}{ccc}
0 & -1 & \varepsilon(t) \sin \gamma_{t} \\
1 & 0 & -\varepsilon(t) \cos \gamma_{t} \\
-\varepsilon(t) \sin \gamma_{t} & \varepsilon(t) \cos \gamma_{t} & 0
\end{array}\right)\left(\begin{array}{c}
x_{t}^{A} \\
y_{t}^{A} \\
z_{t}^{A}
\end{array}\right)
$$

where $\left(x_{0}^{A}, y_{0}^{A}, z_{0}^{A}\right)=\left(\sin \theta_{0} \cos \varphi_{0}, \sin \theta_{0} \sin \varphi_{0}, \cos \theta_{0}\right)$ and $\varphi_{0} \in[0,2 \pi]$. From (53), we have

$$
\left.\dot{z}_{t}\right|_{t=0}=\lim _{\Delta t \rightarrow 0} \frac{z_{\Delta t}^{A}-z_{0}}{\Delta t}=\varepsilon(0) \cos \gamma_{0} y_{0}-\varepsilon(0) \sin \gamma_{0} x_{0}
$$

From (54) and (41), it is easy to obtain the following relationship:

$$
\begin{aligned}
z_{\Delta t}^{A}-z_{\Delta t}^{B}= & z_{0}+\varepsilon(0) \cos \gamma_{0} y_{0} \Delta t-\varepsilon(0) \sin \gamma_{0} x_{0} \Delta t \\
& -z_{0}\left[1-\frac{\varepsilon^{2}(\Delta t)^{2}}{2}\right]+\sin \theta_{0} \varepsilon \Delta t+O\left((\Delta t)^{2}\right) \\
= & \Delta t \sin \theta_{0}\left(\varepsilon+\varepsilon(0) \sin \left(\varphi_{0}-\gamma_{0}\right)\right)+O\left((\Delta t)^{2}\right) .
\end{aligned}
$$

When $\theta_{0} \in[0, \pi), \sin \theta_{0} \geq 0$. Moreover, it is always true that $\varepsilon+\varepsilon(0) \sin \left(\varphi_{0}-\gamma_{0}\right) \geq 0$. If $\theta_{0} \neq 0$ and $\varepsilon+\varepsilon(0) \sin \left(\varphi_{0}-\gamma_{0}\right) \neq 0$, we have

$$
z_{\Delta t}^{A}>z_{\Delta t}^{B}
$$


If $\theta_{0}=0$, we have $\left(x_{0}^{\prime}, y_{0}^{\prime}, z_{0}\right)=\left(x_{0}, y_{0}, z_{0}\right)=(0,0,1)$. According to Lemma 3 and Pontryagin's minimum principle, we have

$$
z_{\Delta t}^{A} \geq z_{\Delta t}^{B}
$$

For $\varepsilon+\varepsilon(0) \sin \left(\varphi_{0}-\gamma_{0}\right)=0$, it must be true that $\varepsilon(0)=\varepsilon$ or $-\varepsilon$. We consider $\varepsilon(0)=\varepsilon$ (for $\varepsilon(0)=-\varepsilon$ we have the same conclusion as $\varepsilon(0)=\varepsilon$ ). Moreover, we have $\gamma_{0}=\varphi_{0}+\frac{\pi}{2}$ or $\gamma_{0}=\varphi_{0}-\frac{3 \pi}{2}$.

For $\gamma_{0}=\varphi_{0}+\frac{\pi}{2}$, we first employ the fact that $z_{t}$ is independent on $\varphi_{0}$ for $H=\varepsilon \sin \varphi_{0} I_{x}-$ $\varepsilon \cos \varphi_{0} I_{y}$ and $\left(x_{0}, y_{0}, z_{0}\right)=\left(\sin \theta_{0} \cos \varphi_{0}, \sin \theta_{0} \sin \varphi_{0}, \cos \theta_{0}\right)$ since $z_{t}=\cos \left(\theta_{0}-\varepsilon t\right)$. Then, it is easy to prove the relationship $z_{\Delta t}^{A} \geq z_{\Delta t}^{B}$ using a similar argument to that in the proof of Lemma 3. For $\gamma_{0}=\varphi_{0}-\frac{3 \pi}{2}$, we have the same conclusion as in the case $\gamma_{0}=\varphi_{0}+\frac{\pi}{2}$. Thus, we obtain

$$
z_{\Delta t}^{A} \geq z_{\Delta t}^{B}
$$

Now using a similar argument to that in the proof of Lemma 5, for arbitrary $t \in\left[0, \frac{\pi}{\varepsilon}\right)$, we have

$$
z_{t}^{A} \geq z_{t}^{B}
$$

From (11), it is clear that the probabilities of failure satisfy $p_{t}^{A}=\frac{1-z_{t}^{A}}{2} \leq p_{t}^{B}=\frac{1-z_{t}^{B}}{2}$. That is, the probability of failure $p_{t}^{A}$ is not greater than $p_{t}^{B}$ for $t \in\left[0, \frac{\pi}{\varepsilon}\right)$. Hence, we can design the measurement period $T$ using the case of $H^{B}$.

Using (11) and (41), for $t \in\left[0, \frac{\pi}{\varepsilon}\right)$, we obtain the probability of failure

$$
p_{t}^{B}=\frac{1-\cos \varepsilon t}{2} .
$$

Hence, we can design the maximum measurement period as follows

$$
T^{(1)}=\frac{\arccos \left(1-2 p_{0}\right)}{\varepsilon} .
$$

\section{CONCLUSIONS}

This paper proposes a variable structure control scheme with sliding modes for the robust control of two-level quantum systems where an eigenstate is identified as a sliding mode. We present a design method for the control laws based on a Lyapunov methodology and periodic projective measurements to drive and maintain this system's state in the sliding mode domain. 
The key task of the control problem is converted into a problem of designing the Lyapunov functions and the measurement period. The Lyapunov function can be constructed to define a control law. By using simulation, we obtain an open-loop control to drive the controlled quantum system's state into the sliding mode domain. For different situations of the uncertainties in the system Hamiltonian, we give two approaches to design the measurement period, which guarantees control performance in the presence of the uncertainties. This sliding mode control scheme provides a robust quantum engineering strategy for controller design for quantum systems and has potential applications in state preparation, decoherence control, quantum error correction [41], etc. Future work which can be carried out in this area is listed as follows. 1) The physical implementation of the proposed method on specific quantum systems. For example, spin systems in NMR (see, e.g., [8], [62]) may be a suitable candidate to test the proposed approach. 2) The extension from two-level systems to multi-level quantum systems: The basic idea of sliding mode control can be extended in a straightforward way to multi-level quantum systems. However, it is much more difficult to obtain an analytical solution for a multi-level system. In [41], a numerical result has been obtained for a three-level quantum system to determine the measurement period and more complex systems are worth exploring by numerical methods. 3) The extension to dissipative quantum systems governed by the Lindblad equation [13] or described by a stochastic differential equation: For such cases, it is necessary to develop new methods to drive the system into the sliding mode domain since the Lyapunov-based control approach does not usually work [63]. 4) The exploration of practical applications for the proposed approaches: The sliding mode may correspond to an eigenstate or a state subspace and the sliding mode design approach could be used in quantum state preparation and protection of encoded quantum information in a subspace.

\section{APPENDIX: PROOF of $T^{(2)} \geq T^{(1)}$}

Proof: Take $p_{0}$ as the variable and define

$$
F\left(p_{0}\right)=T^{(2)}-T^{(1)}
$$

For $p_{0} \in\left(0, \frac{\varepsilon^{2}}{1+\varepsilon^{2}}\right)$, we have the following relationship

$$
f\left(p_{0}\right)=F^{\prime}\left(p_{0}\right)=\frac{1}{\sqrt{\varepsilon^{2} p_{0}-\left(1+\varepsilon^{2}\right) p_{0}^{2}}}-\frac{1}{\sqrt{\varepsilon^{2} p_{0}-\varepsilon^{2} p_{0}^{2}}}
$$


It is clear from (62) and (63) that $f\left(p_{0}\right)>0$ for $p_{0} \in\left(0, \frac{\varepsilon^{2}}{1+\varepsilon^{2}}\right)$ and $F\left(p_{0} \rightarrow 0^{+}\right)=0$. Hence $F\left(p_{0}\right) \geq 0$ for $p_{0} \in\left(0, \frac{\varepsilon^{2}}{1+\varepsilon^{2}}\right)$.

When $p_{0}=p^{\prime}=\frac{\varepsilon^{2}}{1+\varepsilon^{2}}$,

$$
\begin{gathered}
T^{(1)}\left(p^{\prime}\right)=\frac{\arccos \left(\frac{1-\varepsilon^{2}}{1+\varepsilon^{2}}\right)}{\varepsilon}, \\
T^{(2)}\left(p^{\prime}\right)=\frac{\pi}{\sqrt{1+\varepsilon^{2}}} .
\end{gathered}
$$

Let $x=\frac{1-\varepsilon^{2}}{1+\varepsilon^{2}}$ and

$$
G(\varepsilon)=\frac{\varepsilon \pi}{\sqrt{1+\varepsilon^{2}}}-\arccos \left(\frac{1-\varepsilon^{2}}{1+\varepsilon^{2}}\right) .
$$

We have

$$
\widetilde{G}(x)=\frac{\pi}{\sqrt{2}} \sqrt{1-x}-\arccos x .
$$

For $x \in[-1,1], \widetilde{G}(x)$ is continuous in $x$ and we also know that $\widetilde{G}(x)=0$ only when $x= \pm 1$. It is easy to check $\widetilde{G}(x=0)>0$. Hence, we know that for $x \in[-1,1], \widetilde{G}(x) \geq 0$. That is, for $\varepsilon>0, G(\varepsilon) \geq 0$. From the relationship $G(\varepsilon) \geq 0$, we know $T^{(2)}\left(p^{\prime}\right) \geq T^{(1)}\left(p^{\prime}\right)$ for $\varepsilon>0$.

Hence we concluded that for arbitrary $p_{0} \in\left(0, \frac{\varepsilon^{2}}{1+\varepsilon^{2}}\right], T^{(2)} \geq T^{(1)}$.

\section{ACKNOWLEDGMENTS}

The authors would like to thank anonymous reviewers and the Associate Editor for helpful comments and suggestions.

\section{REFERENCES}

[1] D. Dong and I.R. Petersen, "Quantum control theory and applications: A survey," IET Control Theory \& Applications, Vol. 4, pp.2651-2671, 2010.

[2] H.M. Wiseman and G.J. Milburn, Quantum Measurement and Control, Cambridge, England: Cambridge University Press, 2010.

[3] H. Rabitz, "Focus on quantum control", New Journal of Physics, Vol. 11, p.105030, 2009.

[4] S. Chu, "Cold atoms and quantum control", Nature, Vol. 416, pp.206-210, 2002.

[5] H. Rabitz, R. de Vivie-Riedle, M. Motzkus and K. Kompa, "Whither the future of controlling quantum phenomena?" Science, Vol. 288, pp.824-828, 2000.

[6] M.A. Nielsen and I.L. Chuang, Quantum Computation and Quantum Information, Cambridge, England: Cambridge University Press, 2000.

[7] J.P. Dowling and G.J. Milburn, "Quantum technology: the second quantum revolution", Philosophical Transactions of the Royal Society of London A, Vol. 361, pp.1655-1674, 2003. 
[8] N. Khaneja, R. Brockett and S.J. Glaser, “Time optimal control in spin systems”, Physical Review A, Vol. 63, p.032308, 2001.

[9] D. D’Alessandro and M. Dahleh, "Optimal control of two-level quantum systems," IEEE Transactions on Automatic Control, Vol. 46, pp. 866-876, 2001.

[10] S. Grivopoulos and B. Bamieh, "Optimal population transfers in a quantum system for large transfer time," IEEE Transactions on Automatic Control, Vol. 53, pp.980-992, 2008.

[11] W. Cui, Z.R Xi and Y. Pan, "Optimal decoherence control in non-Markovian open dissipative quantum systems", Physical Review A, Vol. 77, p.032117, 2008.

[12] D. Stefanatos, "Optimal design of minimum-energy pulses for Bloch equations in the case of dominant transverse relaxation”, Physical Review A, Vol. 80, p.045401, 2009.

[13] B. Bonnard and D. Sugny, "Time-minimal control of dissipative two-level quantum systems: The integrable case", SIAM Journal on Control and Optimization, Vol. 48, pp.1289-1308, 2009.

[14] U. Boscain and P. Mason, "Time minimal trajectories for a spin 1/2 particle in a magnetic field", Journal of Mathematical Physics, Vol. 47, p.062101, 2006.

[15] R.S. Judson and H. Rabitz, “Teaching lasers to control molecules”, Physical Review Letters, Vol. 68, No. 10, pp.1500-1503, 1992.

[16] H.M. Wiseman and G.J. Milburn, "Quantum theory of optical feedback via homodyne detection", Physical Review Letters, Vol. 70, No. 5, pp.548-551, 1993.

[17] A.C. Doherty, S. Habib, K. Jacobs, H. Mabuchi and S.M. Tan, "Quantum feedback control and classical control theory", Physical Review A, Vol. 62, p.012105, 2000.

[18] B. Qi and L. Guo, "Is measurement-based feedback still better for quantum control systems? " Systems \& Control Letters, Vol. 59, pp.333-339, 2010.

[19] M. Yanagisawa and H. Kimura, “Transfer function approach to quantum control-part I: Dynamics of quantum feedback systems", IEEE Transactions on Automatic Control, Vol. 48, pp.2107-2120, 2003.

[20] R. van Handel, J.K. Stockton and H. Mabuchi, "Feedback control of quantum state reduction", IEEE Transactions on Automatic Control, Vol. 50, No. 6, pp.768-780, 2005.

[21] M. Mirrahimi and R. van Handel, "Stabilizing feedback controls for quantum systems", SIAM Journal on Control and Optimization, Vol. 46, No. 2, pp.445-467, 2007.

[22] N. Yamamoto, K. Tsumura and S. Hara, "Feedback control of quantum entanglement in a two-spin system", Automatica, Vol. 43, pp.981-992, 2007.

[23] V.P. Belavkin, "Measurement, filtering and control in quantum open dynamical systems", Reports on Mathematica Physics, Vol. 43, pp.405-425, 1999.

[24] L. Bouten, R. van Handel and M.R. James, "An introduction to quantum filtering", SIAM Journal on Control and Optimization, Vol. 46, pp.2199-2241, 2007.

[25] F. Ticozzi and L. Viola, "Analysis and synthesis of attractive quantum Markovian dynamics", Automatica, Vol. 45, pp.20022009, 2009.

[26] J. Zhang, R.B. Wu, C.W. Li and T.J. Tarn, "Protecting coherence and entanglement by quantum feedback controls", IEEE Transactions on Automatic Control, Vol. 55, No. 3, pp.619-633, 2010.

[27] H. Mabuchi, "Coherent-feedback quantum control with a dynamic compensator," Physical Review A, Vol. 78, p.032323, 2008 . 
[28] H.I. Nurdin, M.R. James and I.R. Petersen, “Coherent quantum LQG control”, Automatica, Vol. 45, pp.1837-1846, 2009.

[29] G. Zhang and M.R. James, "Direct and indirect couplings in coherent feedback control of linear quantum systems", IEEE Transactions on Automatic Control, Vol. 56, No. 7, pp.1535-1550, 2011.

[30] H.I. Nurdin, M.R. James and A.C. Doherty, "Network synthesis of linear dynamical quantum stochastic systems," SIAM Journal on Control and Optimization, Vol. 48, pp.2686-2718, 2009.

[31] C. D’Helon and M.R. James, "Stability, gain, and robustness in quantum feedback networks", Physical Review A, Vol. 73, p.053803, 2006.

[32] M.R. James, H.I. Nurdin and I.R. Petersen, " $H^{\infty}$ control of linear quantum stochastic systems", IEEE Transactions on Automatic Control, Vol. 53, pp.1787-1803, 2008.

[33] M.A. Pravia, N. Boulant, J. Emerson, E.M. Fortunato, T.F. Havel, D.G. Cory and A. Farid, "Robust control of quantum information", Journal of Chemical Physics, Vol. 119, pp.9993-10001, 2003.

[34] N. Yamamoto, and L. Bouten, "Quantum risk-sensitive estimation and robustness", IEEE Transactions on Automatic Control, Vol. 54, pp.92-107, 2009.

[35] L. Viola and E. Knill, "Robust dynamical decoupling of quantum systems with bounded controls", Physical Review Letters, Vol. 90, p.037901, 2003.

[36] V.I. Utkin, "Variable structure systems with sliding modes", IEEE Transactions on Automatic Control, Vol. AC-22, No. 2, pp.212-222, 1977.

[37] J. Adamy and A. Flemming, "Soft variable structure controls: a survey", Automatica, Vol. 40, pp.1822-1844, 2004.

[38] D. Dong and I.R. Petersen, "Variable structure control of uncontrollable quantum systems", Proc. 6th IFAC Symposium Robust Control Design, p.16, 2009, Haifa, Israel.

[39] D. Dong and I.R. Petersen, "Controllability of quantum systems with switching control", International Journal of Control, Vol. 84, pp.37-46, 2011.

[40] R. Vilela Mendes and V.I. Man'ko, “Quantum control and the Strocchi map”, Physical Review A, Vol. 67, p.053404, 2003.

[41] D. Dong and I.R. Petersen, "Sliding mode control of quantum systems”, New Journal of Physics, Vol. 11, p.105033, 2009.

[42] D. Dong and I.R. Petersen, "Sliding mode control of two-level quantum systems with bounded uncertainties", Proc. of the 2010 American Control Conf., pp.2446-2451, Baltimore, USA, 2010.

[43] M. Mirrahimi, P. Rouchon and G. Turinici, "Lyapunov control of bilinear Schrödinger equations", Automatica, Vol. 41, pp.1987-1994, 2005.

[44] X. Wang and S.G. Schirmer, "Analysis of Lyapunov method for control of quantum states," IEEE Transactions on Automatic Control, Vol. 55, pp. 2259-2270, 2010.

[45] X. Wang and S.G. Schirmer, "Analysis of effectiveness of Lyapunov control for non-generic quantum states," IEEE Transactions on Automatic Control, Vol. 55, pp. 1406-1411, 2010.

[46] S. Grivopoulos and B. Bamieh, "Lyapunov-based control of quantum systems," Proceedings of the 42nd IEEE Conference on Decision and Control, 434-438, Maui, Hawaii USA, Dec. 2003.

[47] C. Altafini, "Feedback stabilization of isospectral control systems on complex flag manifolds: Application to quantum ensembles," IEEE Transactions on Automatic Control, Vol. 52, pp.2019-2028, 2007.

[48] S. Kuang and S. Cong, "Lyapunov control methods of closed quantum systems," Automatica, Vol. 44, pp.98-108, 2008.

[49] B. Misra and E.C.G. Sudarshan, “The Zeno's paradox in quantum theory”, Journal of Mathematical Physics, Vol. 18, pp.756-763, 1977. 
[50] W.M. Itano, D.J. Heinzen, J.J. Bollinger, and D.J. Wineland, “Quantum Zeno effect”, Physical Review A, Vol. 41, pp.22952300, 1990 .

[51] P. Facchi and S. Pascazio, "Quantum Zeno subspaces”, Physical Review Letters, Vol. 89, p.080401, 2002.

[52] F. Ticozzi and S. Bolognani, "On a canonical QR decomposition and feedback control of discrete-time quantum dynamics", Proc. of the 19th International Symposium on Mathematical Theory of Networks and Systems, pp.2065-2071, Budapest, Hungary, July, 2010.

[53] S. Bolognani and F. Ticozzi, "Engineering stable discrete-time quantum dynamics via a canonical QR decomposition", IEEE Transactions on Automatic Control, Vol. 55, pp.2721-2734, 2010.

[54] D. D'Alessandro, Introduction to Quantum Control and Dynamics, Chapman \& Hall/CRC, 2007.

[55] A. Pechen, N. Il'in, F. Shuang and H. Rabitz, "Quantum control by von Neumann measurements", Physical Review A, Vol. 74, p.052102, 2006.

[56] D. Dong, C. Chen, T.J. Tarn, A. Pechen and H. Rabitz, "Incoherent control of quantum systems with wavefunction controllable subspaces via quantum reinforcement learning," IEEE Transactions on Systems Man and Cybernetics-Part B: Cybernetics, Vol. 38, pp.957-962, 2008.

[57] D. Dong, C. Zhang, H. Rabitz, A. Pechen and T.J. Tarn, "Incoherent control of locally controllable quantum systems," Journal of Chemical Physics, Vol. 129, p.154103, 2008.

[58] D. Dong, J. Lam and I.R. Petersen, "Robust incoherent control of qubit systems via switching and optimisation", International Journal of Control, Vol. 83, pp.206-217, 2010.

[59] R. Romano and D. D’Alessandro, "Incoherent control and entanglement for two-dimensional coupled Systems", Physical Review A, Vol. 73, p.022323, 2006.

[60] J. LaSalle and S. Lefschetz, Stability by Liapunov's Direct Method with Applications, New York: Academic Press, 1961.

[61] D.E. Kirk, Optimal Control Theory: An Introduction, Englewood Cliffs, New Jersey: Prentice-Hall Inc., 1970.

[62] J-S. Lee and A.K. Khitrin, "Projective measurement in nuclear magnetic resonance", Applied Physics Letters, Vol. 89, p.074105, 2006.

[63] W. Wang, L.C. Wang and X.X. Yi, "Lyapunov control on quantum open systems in decoherence-free subspaces", Physical Review A, Vol. 82, p.034308, 2010. 